\title{
Angle-deformations in Coxeter groups
}

\author{
TIMOTHÉE MARQUiS \\ BERNHARD MÜHLHERR
}

\begin{abstract}
The isomorphism problem for Coxeter groups has been reduced to its "reflection preserving version" by B Howlett and the second author. Thus, in order to solve it, it suffices to determine for a given Coxeter system $(W, R)$ all Coxeter generating sets $S$ of $W$ which are contained in $R^{W}$, the set of reflections of $(W, R)$. In this paper, we provide a further reduction: it suffices to determine all Coxeter generating sets $S \subseteq R^{W}$ which are sharp-angled with respect to $R$.
\end{abstract}

20F55; 51F15

\section{Introduction}

Let $W$ be a group and let $R \subseteq W$. We call $R$ a Coxeter generating set of $W$ if $(W, R)$ is a Coxeter system. All Coxeter systems $(W, R)$ considered in this paper are assumed to have finite rank, ie $R$ is a finite set.

Let $(W, R)$ be a Coxeter system and let $S \subseteq R^{W}$ be a Coxeter generating set of $W$. A subset $J$ of $S$ is called spherical if it generates a finite subgroup; if it is of cardinality 2, it is called an edge of $S$. Let $\{s, t\} \subseteq S$ be an edge of $S$. By basic results on Coxeter groups, one knows that there exist $r, r^{\prime} \in R$ and $w \in W$ such that $\langle s, t\rangle^{w}=\left\langle r, r^{\prime}\right\rangle$. If there exist $r, r^{\prime} \in R$ and $w \in W$ such that $\{s, t\}^{w}=\left\{r, r^{\prime}\right\}$, then we call the edge $\{s, t\}$ sharp-angled with respect to $R$. We call $S$ sharp-angled with respect to $R$ if all edges of $S$ are sharp-angled with respect to $R$. The trivial example of the dihedral groups shows that there are examples of Coxeter systems $(W, R)$ admitting Coxeter generating sets $S \subseteq R^{W}$ which are not sharp-angled with respect to $R$.

In [11], the second author conjectured that for any Coxeter generating set $S \subseteq R^{W}$, there exists an automorphism $\alpha$ of $W$ such that $\alpha(S) \subseteq R^{W}$ and such that $\alpha(S)$ is sharp-angled [11, Conjecture 1]. This conjecture may be seen as a reduction step in order to state the main conjecture about the solution of the isomorphism problem for Coxeter groups, which is Conjecture 2 in [11] (see Remark 1 below).

It was mentioned without proof in [11] that Conjecture 1 is true if there is no subdiagram of type $\mathrm{H}_{3}$. It turned out that this conjecture was too optimistic if there are 
$\mathrm{H}_{3}$-subdiagrams. Counterexamples have been found independently by Ratcliffe and Tschantz [12] and by Grassi [9]. This motivates the question whether it is still true that one can reduce the solution for the isomorphism problem to the main conjecture. The goal of this paper is to show that this is indeed the case.

Our first result is the following.

Theorem 1 Let $(W, R)$ be a Coxeter system. Let $S \subseteq R^{W}$ be a Coxeter generating set of $W$ having no subsystem of type $H_{3}$. Then there exists an automorphism $\alpha$ of $W$ such that $\alpha(S)$ is sharp-angled with respect to $R$.

As already mentioned before, Theorem 1 was announced in [11] and it is a special case of Theorem 2 below. Its proof is given in Section 6. We prefer to present it separately since it is rather easy and provides at the same time a good overview on the kind of arguments that will yield Theorem 2 .

The situation becomes considerably more complicated if $H_{3}$-subdiagrams are allowed. First of all, the counterexamples to [11, Conjecture 1] show that one cannot expect to produce sharp-angled Coxeter generating sets from $S$ by automorphisms. So, we have to produce the desired Coxeter generating set starting from $S$ by a sequence of operations which we call angle-deformations.

In order to define angle-deformations, we analyse the situation where we are given a Coxeter system $(W, R)$ and a Coxeter generating set $S \subseteq R^{W}$ such that there is an edge $J$ of $S$ which is not sharp-angled with respect to $R$. It turns out that the Coxeter diagram of the system $(W, S)$ has to satisfy several conditions with respect to the subset $J$. These conditions will be deduced in Section 8. An edge satisfying these conditions will be called a $\Delta-e d g e$.

Let $(W, S)$ be a Coxeter system and $J=\{r, s\}$ be a $\Delta$-edge of $S$. Then we construct a mapping $\delta: S \rightarrow W$ such that $\delta(s)=s, \delta(r) \in\langle s, r\rangle$ and such that $S^{\prime}:=\{\delta(x) \mid$ $x \in S\}$ is a Coxeter generating set with the property that all spherical 2-subsets $\left\{x^{\prime}, y^{\prime}\right\} \neq\{\delta(r), \delta(s)\}$ are sharp-angled with respect to $S$. We call these mappings $J$-deformations. In the case where there are no $H_{3}$-subdiagrams, it is easy to give the definition of these $\mathrm{J}$-deformations. If there are $\mathrm{H}_{3}$-subdiagrams, the definition is given recursively. We first define $J$-deformations for a class of diagrams which we call tame. The general case will then be treated by induction on the number of "wild" vertices.

The construction of $J$-deformations will enable us to prove our main result, which is the following. 
Theorem 2 Let $(W, R)$ be a Coxeter system and let $S \subseteq R^{W}$ be a Coxeter generating set of $W$. Then there exists a sequence $S=S_{0}, \ldots, S_{k}=S^{\prime}$ of Coxeter generating sets $S_{i}$ such that $S_{i}$ is a $J_{i}$-deformation of $S_{i-1}$ for some $\Delta$-edge $J_{i}$ of $S_{i-1}$ for each $1 \leq i \leq k$, and such that $S^{\prime}$ is sharp-angled with respect to $R$.

We remark that the proof of Theorem 2 is constructive. Hence it provides a concrete algorithm to obtain the set $S^{\prime}$ starting from $S$. Combining the theorem above with the fact that the isomorphism problem for Coxeter groups is reduced to its "reflectionpreserving version" (as described in [11]), we obtain the following.

Corollary 1.1 The isomorphism problem for Coxeter groups is solved as soon as the following problem is solved.

Problem Let $(W, R)$ be a Coxeter system. Find all Coxeter generating sets $S \subset R^{W}$ such that $S$ is sharp-angled with respect to $R$.

Remark 1 There is a conjecture about the solution of the above problem. This is Conjecture 2 in [11] and it is a refinement of Conjecture 8.1 in Brady-McCammondMühlherr-Neumann [2]. It says that if $R$ and $S$ are as in the problem above, one can transform $S$ into $R$ by a sequence of twists introduced in [2]. The conjecture has been proved for various classes of Coxeter systems; the reader may refer to [11] for a survey on its status in 2005. Recently, it was shown by Ratcliffe and Tschantz in [12] that the conjecture holds for chordal Coxeter systems as well.

Remark 2 In [12], Ratcliffe and Tschantz obtained Theorem 2 for chordal Coxeter systems. Their methods are quite different from ours. Their arguments rely heavily on a very strong property of chordal Coxeter groups which is not available in the general case.

The paper is organized as follows. In Section 2, we fix notation, recall some basic facts on Coxeter groups and provide some preliminary results. In Section 3, we introduce angle-deformations and make some observations about them. In Section 4, we prepare the proof of Theorem 1. In this section we introduce $\Theta$-edges, which are special cases of $\Delta$-edges. Section 5 is devoted to introducing and investigating the notion of a sharp-angled set of reflections in a Coxeter group. This will enable us to give the proof of Theorem 1 in Section 6. In Section 7, we collect information about angledeformations of Coxeter systems with subdiagrams of type $H_{3}$ and $H_{4}$. In Section 8, we define $\Delta$-edges. Later on, these turn out to be precisely the edges of a Coxeter system for which there are nontrivial angle-deformations. This fact is a consequence 
of Proposition 6.1 and Theorem 10.4, and it is indeed the key ingredient of the proof of our main result. Section 9 can be seen as a preparatory section for the proof of Theorem 10.4 which will be completed in Section 10. In Section 11, we finally give the proof of our main result, Theorem 2.

\section{Preliminaries}

\subsection{Graphs}

For a set $X$, denote by $P_{2}(X)$ the set of all subsets of $X$ having cardinality 2. A graph is a pair $(V, E)$ consisting of a set $V$ and a set $E \subseteq P_{2}(V)$. The elements of $V$ and $E$ are called vertices and edges respectively.

Let $\Gamma=(V, E)$ be a graph. Let $v, w$ be two vertices of $\Gamma$. They are called adjacent if $\{v, w\} \in E$. In this paper, a path from $v$ to $w$ is a sequence $v=v_{0}, v_{1}, \ldots, v_{k}=w$, where $v_{i-1}$ is adjacent to $v_{i}$ for all $1 \leq i \leq k$ and where $v_{1}, \ldots, v_{k}$ are pairwise distinct; the number $k$ is the length of the path. The path is minimal if it is of minimal length. The distance between $v$ and $w$ (denoted by $\delta(v, w)$ ) is the length of a minimal path joining them; if there is no path joining $v$ and $w$, we put $\delta(v, w)=\infty$.

A path $v=v_{0}, v_{1}, \ldots, v_{k}=w$ is said to be chordfree if $E \cap P_{2}\left(\left\{v_{0}, \ldots, v_{k}\right\}\right)=$ $\left\{\left\{v_{0}, v_{1}\right\},\left\{v_{1}, v_{2}\right\}, \ldots,\left\{v_{k-1}, v_{k}\right\}\right\}$. A path $v=v_{0}, v_{1}, \ldots, v_{k}=w$ is called a circuit if $v=w$ and $k \geq 2$.

The relation $R \subseteq V \times V$ defined by $R=\{(v, w) \mid \delta(v, w) \neq \infty\}$ is an equivalence relation whose equivalence classes are called the connected components of $\Gamma$. A graph is said to be connected if it has only one connected component.

\subsection{Coxeter systems}

Let $(W, S)$ be a pair consisting of a group $W$ and a set $S \subseteq W$ of involutions. For $r, s \in S$, denote by $m_{r s} \in \mathbb{N} \cup\{\infty\}$ the order of the product $r s$ in $W$. Note that we will also use the notation $o(r s)$ instead of $m_{r s}$. Define $E(S):=\left\{\{r, s\} \subseteq S \mid 1 \neq m_{r s} \neq \infty\right\}$ to be the set of edges of $S$. Then $\Gamma(S)$ is the graph $(S, E(S))$ whose edges are labelled by the corresponding $m_{r s}$. Throughout this text, any graph notion (such as paths and circuits) associated to the pair $(W, S)$ must be understood as being in $\Gamma(S)$. In particular, when we speak about the "diagram of $(W, S)$ ", we refer to $\Gamma(S)$.

The Coxeter diagram associated to $(W, S)$ is the graph $\left(S, E^{\prime}(S)\right)$ where $E^{\prime}(S):=$ $\left\{\{r, s\} \subseteq S \mid m_{r s} \geq 3\right\}$ and where the edges are labelled by the corresponding $m_{r s}$. A subset $K$ of $S$ is said to be irreducible if the underlying Coxeter subdiagram 
$\left(K, E^{\prime}(K)\right)$ is connected. We call $K$ spherical if it generates a finite group. Finally, $K$ is 2-spherical if $m_{r s}<\infty$ for all $r, s \in K$. If $S$ is irreducible, spherical or 2-spherical, we say that $(W, S)$ is irreducible, spherical or 2-spherical, respectively. Note that sometimes, we use the same notions for $\Gamma(S)$ instead of $(W, S)$.

We say that $(W, S)$ is a Coxeter system if $S$ generates $W$ and if the relations $\left((r s)^{m_{r s}}\right)_{r, s \in S}$ form a presentation of $W$. We call $R \subseteq W$ a Coxeter generating set if $(W, R)$ is a Coxeter system.

Let $(W, R)$ be a Coxeter system. An element of $W$ is called a reflection if it is conjugate in $W$ to an element of $R$; the set of all reflections is denoted by $R^{W}$.

\subsection{Conventions about figures}

Here are some conventions about the figures appearing in the paper, which the reader may refer back to when needed.

Throughout this text, all figures represent diagrams of the form $\Gamma(K)$ for some Coxeter system $(W, S)$ and $K \subseteq S$. The edges in plain have a finite label, while the edges in strips have an infinite label. An absence of edge does not imply anything. If there is a single edge with more than one label (say $m>1$ ), then the figure must be understood as $m$ different figures, one for each of these labels. If there are two or more edges with more than one label, then these edges will have the same number $m>1$ of labels. In that case, the figure must be understood as $m$ different figures, the $i$-th figure being obtained by taking the $i$-th label from each of these edges.

A dotted line between two vertices means that there is a path (in plain) joining these two vertices, but the other vertices in the path were omitted. (It will be always clear from the context what the omitted vertices are). For example, in Section 8, Figure 2 and Figure 3 contain a path $\{S(1), S(2), \ldots, S(n-1), S(n)\}$. We denote by $X$ this set and we assume $n \geq 2$. Let $X_{1}:=X \backslash\{S(1)\}$ and $X_{n}:=X \backslash\{S(n)\}$. We assume $X$ has the following property:

$$
o(S(i) S(j))=\infty \text { for all } i, j \text { such that } 1 \leq i<j \leq n \text { and }|i-j| \geq 2 .
$$

Finally, for a vertex $y \notin X_{1}$, we mean by $\overline{X_{1} y}=\infty$ that $m_{x y}=\infty$ for all $x \in X_{1}$.

\subsection{Coxeter generating sets and automorphisms}

Lemma 2.1 Let $(W, S)$ be a Coxeter system and let $S_{1}, S_{2}$ be subsets of $S$ such that each edge of $S$ is contained in $S_{1}$ or $S_{2}$. Put $S_{0}:=S_{1} \cap S_{2}$. Let $\delta: S \rightarrow W$ be a mapping such that $\delta\left(S_{i}\right)$ is a Coxeter generating set of $\left\langle S_{i}\right\rangle$ for $i=0,1,2$. Then $\delta(S)$ 
is a Coxeter generating set of $W$. Moreover, if the restrictions of $\delta$ to $S_{1}$ and $S_{2}$ extend to automorphisms of $\left\langle S_{1}\right\rangle$ and $\left\langle S_{2}\right\rangle$ respectively, then $\delta$ extends to an automorphism of $W$.

Proof This follows immediately from the fact that $W=\left\langle S_{1}\right\rangle *\left\langle S_{0}\right\rangle\left\langle S_{2}\right\rangle$.

The following lemma follows easily by the pigeon-hole principle.

Lemma 2.2 Let $G$ be a finite group, let $\alpha$ be an automorphism of $G$ and let $g \in G$. Then $\alpha^{m}(g) \alpha^{m-1}(g) \ldots \alpha^{2}(g) \alpha(g) g=1_{G}$ for some $m \geq 0$.

Using the previous lemma, one immediately obtains the following proposition.

Proposition 2.3 Let $(W, S)$ be a Coxeter system and let $\alpha: W \rightarrow W$ be an epimorphism. Suppose that there is a subset $\mathbf{K}$ of $2^{S}$ such that the following holds:

(1) All elements of $\mathbf{K}$ are spherical.

(2) For all $K \in \mathbf{K}$, the mapping $\left.\alpha\right|_{\langle K\rangle}$ is an automorphism of $\langle K\rangle$.

(3) For all $s \in S$, there exists $w_{s} \in \bigcup_{K \in \mathbf{K}}\langle K\rangle$ such that $\alpha(s)=w_{s} s w_{s}^{-1}$.

Then $\alpha$ is an automorphism of $W$ which is of finite order.

\subsection{The geometric representation of a Coxeter system}

In this subsection, we collect several basic results about the geometric representation of a Coxeter system. The standard references are Bourbaki [1] and Humphreys [10].

Throughout this paper, $\Omega$ and $\Omega^{\prime}$ are the following subsets of $\mathbb{R}$ :

$$
\Omega:=\{\cos (\pi / m) \mid m \in \mathbb{N}\} \cup[1, \infty)
$$

and $\Omega^{\prime}:=\Omega \backslash\{-1\}$. Moreover, we define a mapping $C: \mathbb{N} \cup\{\infty\} \rightarrow-\Omega$ by setting $C(m):=-\cos (\pi / m)$ if $m \in \mathbf{N}$ and $C(\infty):=-1$.

Let $V$ be a real vector space endowed with a symmetric bilinear form $b: V \times V \rightarrow \mathbb{R}$. The set of vectors $v \in V$ with $b(v, v)=1$ is denoted by $U(V, b)$ and for each such vector, the corresponding orthogonal reflection with respect to $b$ is denoted by $\rho_{v}$; hence $\rho_{v}(x)=x-2 b(x, v) v$ for each $x \in V$.

Let $(W, R)$ be a Coxeter system. Let $V:=\mathbb{R}^{R}$ and $\left(e_{r}\right)_{r \in R}$ be the canonical basis of $V$. Furthermore, let $b: V \times V \rightarrow \mathbb{R}$ be the symmetric bilinear form defined by $b\left(e_{r}, e_{s}\right):=C(o(r s))$. 
Theorem 2.4 The mapping $r \mapsto \rho_{e_{r}}$ from $R$ into $O(V, b)$ extends to a monomorphism from $W$ into $O(V, b)$.

Thus, by the above construction, we obtain a canonical faithful linear representation of the Coxeter group $W$ which is called the geometric representation of $(W, R)$. We now identify $W$ with its image in $O(V, b)$ and we put $\Phi(W, R):=\left\{w\left(e_{r}\right) \mid w \in W, r \in R\right\}$. We have the following:

Lemma 2.5 For all $r \in R$ and $w \in W$, we have $\rho_{w\left(e_{r}\right)}=w r w^{-1}$; in particular, $R^{W}=\left\{\rho_{\alpha} \mid \alpha \in \Phi(W, R)\right\}$. Moreover, if $\alpha, \beta \in \Phi(W, R)$ are such that $\rho_{\alpha}=\rho_{\beta}$, then $\beta=\alpha$ or $\beta=-\alpha$.

The set $\Phi:=\Phi(W, R)$ is called the root system of $(W, R)$. We put

$$
V^{+}:=\left\{\Sigma_{r \in R} \mu_{r} e_{r} \mid \mu_{r} \geq 0 \text { for all } r \in R\right\}
$$

and $V^{-}:=-V^{+}$; furthermore, we put $\Phi^{+}:=V^{+} \cap \Phi$ and $\Phi^{-}:=V^{-} \cap \Phi$.

Lemma 2.6 $\Phi=\Phi^{+} \cup \Phi^{-}$.

The elements of $\Phi^{+}$are called the positive roots of $(W, R)$. A subset $\Pi$ of $\Phi$ is called a root-subbase of $\Phi$ if $\Pi \subseteq \Phi^{+}$and if $b(\alpha, \beta) \in-\Omega^{\prime}$ for all $\alpha \neq \beta \in \Pi$.

The following theorem is a consequence of the main result in Deodhar [6] and Dyer [7].

Theorem 2.7 Let $\Pi$ be a root subbase of $\Phi$ and put $S:=\left\{\rho_{\alpha} \mid \alpha \in \Pi\right\}$. Then $(\langle S\rangle, S)$ is a Coxeter system. Conversely, let $W^{\prime}$ be a subgroup of $W$ which is generated by a set of reflections. Then there exists a root-subbase $\Pi^{\prime}$ of $\Phi$ such that $W^{\prime}=\left\langle\rho_{\alpha} \mid \alpha \in \Pi^{\prime}\right\rangle$.

\subsection{Flexibility}

Let $(W, S)$ be a Coxeter system and $J \subseteq S$. We define the following notions and notation:

- $J^{\perp}:=\left\{s \in S \mid \forall j \in J: m_{s j}=2\right\}$.

- $J^{\text {fin }}:=\left\{s \in S \backslash J \mid m_{s j}<\infty \forall j \in J\right\}$.

- $J^{\infty}:=\left\{s \in S \backslash J \mid \exists j \in J: m_{s j}=\infty\right\}$.

- $\mathcal{G}_{J}:=\left(J^{\infty},\left\{\{a, b\} \subseteq J^{\infty} \mid m_{a b}<\infty\right\}\right)$.

- A $J$-component is a connected component of $\mathcal{G}_{J}$. 
- Let $L$ be a $J$-component. We shall say that $j \in J$ is $L$-free if $m_{j l}=\infty$ for all $l \in L$.

- An element $j$ of $J$ is $J^{\infty}$-free if it is $L$-free for every $J$-component $L$.

- The $J$-component $L$ is said to be flexible if there exists $j \in J$ such that $j$ is $L-$ free.

- Finally, we will say that $J$ is flexible if all $J$-components are.

Here is a first observation.

Lemma 2.8 Let $(W, S)$ be a Coxeter system and let $J=\{r, s\}$ be an edge of $S$. Then $J$ is flexible if and only if there is no chordfree circuit in $\Gamma(S)$ of length at least 4 containing $J$.

Proof Suppose first $J$ is not flexible. Then there exists a $J$-component $L$ and $x, y \in L$ such that $m_{x r}<\infty$ and $m_{y s}<\infty$. Let $x=x_{0}, x_{1}, \ldots, x_{k}=y$ be a minimal path in $L$ joining $x$ to $y$. Define

and

$$
\begin{aligned}
M & :=\min \left\{i \mid 0<i \leq k ; m_{x_{i} s}<\infty\right\} \\
m & :=\max \left\{i \mid 0 \leq i<M ; m_{x_{i} r}<\infty\right\} .
\end{aligned}
$$

Then the subpath $x_{m}, x_{m+1}, \ldots, x_{M}$ from $x_{m}$ to $x_{M}$ is still minimal, hence chordfree, and possesses the following properties:

(1) $m_{x_{i} s}=\infty$ for all $i$ such that $m \leq i<M$ (by definition of $\mathrm{M}$ ).

(2) $m_{x_{i} r}=\infty$ for all $i$ such that $m<i \leq M$ (by definition of $\mathrm{m}$ ).

Moreover, $m_{x_{M} s}<\infty$ and $m_{x_{m} r}<\infty$. We then obtain a chordfree circuit $r, x_{m}$, $x_{m+1}, \ldots, x_{M}, s, r$, as required. The situation is illustrated on Figure 1.

The converse is obvious.

\section{Angle-deformations}

Definition 3.1 Let $(W, S)$ be a Coxeter system, let $J=\{r, s\}$ be an edge of $S$ and let $\omega \in\langle J\rangle$ be such that $\omega r \omega^{-1}$ and $s$ generate $\langle J\rangle$. An $(r, s, \omega)$-deformation of $S$ is a mapping $\delta: S \rightarrow W$ satisfying the following properties:

(AD1) $\delta(x) \in S^{W}$ for all $x \in S$.

(AD2) $\delta(r)=\omega r \omega^{-1}$ and $\delta(s)=s$. 


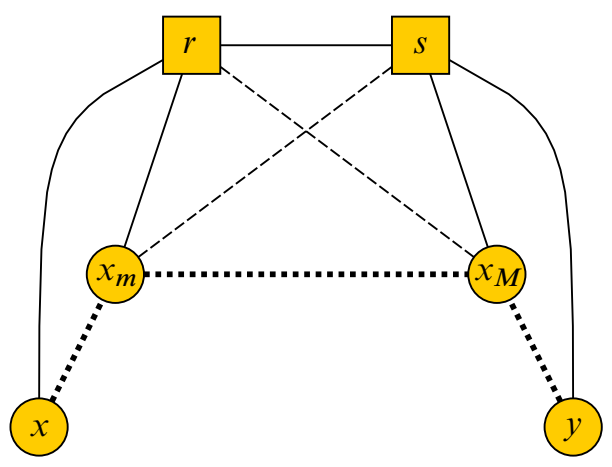

Figure 1. Lemma 2.8

(AD3) $\delta(S)$ is a Coxeter generating set of $W$.

(AD4) There exists a bijection $\Delta$ from the set of edges of $S$ onto the set of edges of $\delta(S)$ such that $\Delta(J)=\left\{\omega r \omega^{-1}, s\right\}$ and such that for each edge $K \neq J$ of $S$, there exists $w_{K} \in W$ with $\Delta(K)=K^{w_{K}}$.

Definition 3.2 Let $(W, S)$ be a Coxeter system and let $J=\{r, s\}$ be an edge of $S$. A $J$-deformation of $S$ is an $(r, s, \omega)$-deformation of $S$ for some $\omega \in\langle J\rangle$. An angle-deformation of $S$ is a $J$-deformation for some edge $J$ of $S$.

The following proposition is a consequence of Lemma 2.1 .

Proposition 3.3 Let $(W, S)$ be a Coxeter system and let $S_{1}, S_{2}$ be subsets of $S$ such that each edge of $S$ is contained in $S_{1}$ or $S_{2}$ and put $S_{0}:=S_{1} \cap S_{2}$. Let $J$ be an edge contained in $S_{0}$ and assume that $\delta_{i}: S_{i} \rightarrow\left\langle S_{i}\right\rangle$ are $J$-deformations of $S_{i}$ for $i=0,1,2$ and that $\delta_{0}=\left.\delta_{i}\right|_{S_{0}}$ for $i=1,2$. Define $\delta: S_{1} \cup S_{2} \rightarrow\left\langle S_{1} \cup S_{2}\right\rangle$ by setting $\left.\delta\right|_{S_{i}}:=\delta_{i}$ for $i=1,2$. Then $\delta$ is a $J$-deformation of $S_{1} \cup S_{2}$.

Proposition 3.4 Let $(W, S)$ be a Coxeter system, $J:=\{r, s\}$ be an edge of $S$ and let $\omega \in\langle J\rangle$ be such that $\omega r \omega^{-1}$ and $s$ generate $\langle J\rangle$. Let $\mathbf{K}$ be a set of spherical subsets of $S$ such that each element of $\mathbf{K}$ contains $J$ and let $\delta: S \rightarrow W$ be a mapping with the following properties:

a) $\delta(r)=\omega r \omega^{-1}$ and $\delta(s)=s$.

b) $\langle\delta(S)\rangle=W$.

c) For all $x \in S$, there exists an element $w_{x}$ in $\bigcup_{K \in \mathbf{K}}\langle K\rangle$ such that $\delta(x)=$ $w_{x} x w_{x}^{-1}$. 
d) For each edge $E$ of $S$ different from $J$, there exists an element $w_{E} \in W$ such that $\delta(E)=E^{w_{E}}$.

Then $\delta$ is an $(r, s, \omega)$-deformation of $S$ which extends to an automorphism of $W$.

Proof By the universal property of $(W, S)$ and Property d), $\delta$ extends to an endomorphism $\alpha$ of $W$ which is in fact an epimorphism because of Property b). By Proposition 2.3 , it follows now from Property c) that $\alpha$ is an automorphism. Hence $\delta(S)$ is a Coxeter generating set of $W$ and the mapping $E \mapsto \delta(E)$ is a bijection as required in Condition (AD4). As (AD1) is a consequence of Property c), and as (AD2) is precisely Property a), the proposition is proved.

\section{Angle-deformations involving dihedral groups}

Throughout this section, $(W, S)$ is a Coxeter system and $J=\{r, s\}$ is an edge of $S$ such that $o(r s) \geq 3$.

\subsection{Condition (TWa)}

Definition 4.1 Let $a \in J$. We say that $J$ is an $a$-special subset of $S$ if the following condition (TWa) holds.

(TWa) For all $x \in S \backslash J$ we have $o(x a) \in\{2, \infty\}$, and if $o(x a)=2$ then $x \in J^{\perp}$.

The following observation is immediate.

Lemma 4.2 Let $a \in J$ be such that $J$ is $a$-special. Then the following holds.

a) $\left\{J, J^{\infty}, J^{\perp}\right\}$ is a partition of $S$.

b) $a$ is $J^{\infty}$-free; in particular, $J$ is flexible.

For the remainder of this subsection, we assume that $a \in J$ is such that $J$ is $a$-special, and $\omega \in\langle J\rangle$ is such that $\omega r \omega^{-1}$ and $s$ generate $\langle J\rangle$. We put $\pi:=1_{W}$ if $a=r$ and $\pi:=\omega$ if $a=s$. Moreover, we let $\delta: S \rightarrow W$ be the mapping defined by $\delta(r)=\omega r \omega^{-1}, \delta(y)=y$ for $y \in\{s\} \cup J^{\perp}$ and $\delta(x):=\pi x \pi^{-1}$ if $x \in J^{\infty}$.

Lemma 4.3 Let $E=\{x, y\}$ be an edge of $S$ different from $J$. Then there exists $w_{E}$ such that $\delta(E)=E^{w_{E}}$. 
Proof Note first that each $y \in J^{\perp}$ commutes with $\omega$ and $\pi$. Hence, if $E \subseteq\{s\} \cup J^{\perp}$, then we may choose $w_{E}=1_{W}$; if $E \subseteq\{r\} \cup J^{\perp}$, then we may choose $w_{E}=\omega$; and if $E \subseteq J^{\infty} \cup J^{\perp}$, then we may choose $w_{E}=\pi$.

By the previous lemma, we are left with the case where $E \subseteq J \cup J^{\infty}$. As $a$ is $J^{\infty}$-free and $E \neq J$, we are now left with the case where $E=\{b, x\}$ for some $x \in J^{\infty}$ and where $b$ is the element of $J$ distinct from $a$. If $a=r$, we may choose $w_{E}=1_{W}$ and if $a=s$, we may choose $w_{E}=\omega$.

Proposition 4.4 The mapping $\delta$ is an $(r, s, \omega)$-deformation of $S$ which extends to an automorphism of $W$.

Proof Setting $\mathbf{K}:=\{J\}$, Properties a), b) and c) required in Proposition 3.4 are clear from the definition of $\delta$ and Property d) is settled by the previous lemma.

\section{$4.2 \Theta$-edges}

Definition 4.5 We say that $J$ is a $\Theta-e d g e$ of $S$ if $J$ is flexible and if there is no 2 -spherical and irreducible subset of $S$ containing $J$ properly.

Remark If $J$ is a $\Theta$-edge, then $\left\{J, J^{\infty}, J^{\perp}\right\}$ is a partition of $S$.

For the remainder of this subsection, we suppose that $J$ is a $\Theta$-edge of $S$. Moreover, we assume that $\omega \in\langle J\rangle$ is such that $\omega r \omega^{-1}$ and $s$ generate $\langle J\rangle$.

Let $L$ be a $J$-component. We denote the set of $L$-free vertices in $J$ by $\Pi(L)$. It is nonempty because $J$ is assumed to be flexible. If $r \in \Pi(L)$, we put $a_{L}:=r$ and $\gamma_{L}:=1_{W}$; if this is not the case, we set $a_{L}:=s$ and $\gamma_{L}:=\omega$. We set $K_{L}:=J \cup L \cup J^{\perp}$. We define $\delta_{L}: K_{L} \rightarrow\left\langle K_{L}\right\rangle$ by $\delta_{L}(r):=\omega r \omega^{-1}, \delta_{L}(y):=y$ for all $y \in\{s\} \cup J^{\perp}$ and $\delta_{L}(x):=\gamma_{L} x \gamma_{L}^{-1}$ for all $x \in L$.

Proposition 4.6 Let $\delta: S \rightarrow W$ be the unique mapping such that $\left.\delta\right|_{K_{L}}=\delta_{L}$ for every $J$-component $L$. Then $\delta$ is an $(r, s, \omega)$-deformation of $S$ which extends to an automorphism of $W$.

Proof Let $L$ be a $J$-component. The edge $J$ is an $a_{L}$-special subset of $K_{L}$ and hence it follows by Proposition 4.4 that $\delta_{L}$ is an $(r, s, \omega)$-deformation of $K_{L}$. An obvious induction on the number of $J$-components using Proposition 3.3 and Lemma 2.1 yields the claim. 


\section{Sharp-angled sets of reflections}

Throughout this section, $(W, R)$ denotes a Coxeter system, where $W$ is identified with its image in $O(V, b)$ by its geometric representation and $\Phi \subseteq U(V, b)$ is its root system.

Lemma 5.1 Let $\alpha, \beta \in \Phi$.

a) If $|b(\alpha, \beta)|<1$, then $o\left(\rho_{\alpha} \rho_{\beta}\right)$ is finite and $b(\alpha, \beta)=-\cos (p \pi / q)$ for some integers $p$ and $q$.

b) If $\rho_{\alpha} \neq \rho_{\beta}$ and $|b(\alpha, \beta)| \geq 1$, then $\rho_{\alpha} \rho_{\beta}$ has infinite order.

c) If $\rho_{\alpha} \neq \rho_{\beta}$, then $o\left(\rho_{\alpha} \rho_{\beta}\right)$ is finite if and only if $|b(\alpha, \beta)|<1$.

Proof Assertion a) is Proposition 1.4 in Brink and Howlett [3], whereas Assertion b) is an easy exercise in linear algebra. Assertion c) is an immediate consequence of a) and $b$ ).

Definition 5.2 Let $s \neq t \in R^{W}$ be such that $o(s t)$ is finite. Let $\alpha, \beta \in \Phi$ be such that $s=\rho_{\alpha}$ and $t=\rho_{\beta}$. Then we call the 2-set $\{s, t\}$ sharp-angled if $|b(\alpha, \beta)| \in \Omega$.

Remark Note that this definition does not depend on the choice of $\alpha$ and $\beta$ in view of the last statement of Lemma 2.5.

The following two lemmas are easy.

Lemma 5.3 Let $s \neq r \in R^{W}$ be such that $o(r s)$ is finite. If $\{r, s\}$ is not sharp-angled, then $o(r s) \geq 5$.

Lemma 5.4 Let $s \neq r \in R^{W}$ be such that $o(r s)$ is finite and suppose $\{r, s\}$ is not sharp-angled. Then there exists an element $w \in\langle s, r\rangle$ such that the set $\left\{s, w r w^{-1}\right\}$ is sharp-angled. Moreover, if $o(r s)=5$, we may choose $w$ to be $s r s$.

Definition 5.5 A set $S \subset R^{W}$ is called sharp-angled if each edge of $S$ is sharp-angled.

The following lemma follows from the fact that $W$ is a subgroup of $O(V, b)$ and from the first statement of Lemma 2.5.

Lemma 5.6 Let $S$ be a set of reflections and let $w \in W$. Then $S^{w}$ is sharp-angled if and only if $S$ is sharp-angled.

The following fact follows from the definition of a root-subbase:

Lemma 5.7 Let $\Pi$ be a root-subbase of $\Phi$ and $S:=\left\{\rho_{\alpha} \mid \alpha \in \Pi\right\}$. Then $S$ is sharp-angled. 


\subsection{Fundamental sets of reflections}

Definition 5.8 A subset $S$ of $R^{W}$ is called fundamental if $(\langle S\rangle, S)$ is a Coxeter system.

Theorem 5.9 Let $S \subset R^{W}$ be a fundamental set of reflections and suppose that one of the following holds:

A) The Coxeter system $(\langle S\rangle, S)$ is 2-spherical, irreducible and nonspherical.

B) $\Gamma(S)$ is a chordfree circuit of length at least 4 .

Then $S$ is sharp-angled.

Proof As $W^{\prime}:=\langle S\rangle$ is generated by a set of reflections, we may apply the second part of Theorem 2.7 to see that there is a root-subbase $\Pi$ of $\Phi$ such that the set $S^{\prime}:=\left\{\rho_{\alpha} \mid \alpha \in \Pi\right\}$ is a Coxeter generating set of $W^{\prime}$. It is known by CapraceMühlherr [4] and Charney-Davis [5] that the Coxeter system $(\langle S\rangle, S)$ is strongly reflection rigid and hence $S$ and $S^{\prime}$ are conjugate in $W^{\prime}$ and the claim follows from Lemma 5.6 and Lemma 5.7.

\section{Proof of Theorem 1}

Throughout this section, $(W, R)$ is a Coxeter system and $S \subseteq R^{W}$ is a fundamental set of reflections. Moreover, we assume that $S$ contains no subset of type $\mathrm{H}_{3}$.

Proposition 6.1 Suppose that $J$ is an edge of $S$ which is not sharp-angled. Then $J$ is a $\Theta$-edge of $S$.

Proof Put $J=\{r, s\}$. By Lemma 5.3, we have $o(r s) \geq 5$. Let $t \in S$ be such that $o(r t)$ and $o(s t)$ are finite. By Theorem 5.9 and our hypothesis that there are no subsets of type $H_{3}$, we have that $t \in J^{\perp}$. Hence there is no irreducible 2-spherical subset of $S$ containing $J$ properly. Furthermore, again by Theorem 5.9, there is no chordfree circuit of length at least 4 containing $J$. By Lemma 2.8, it follows that $J$ is flexible. Hence $J$ is indeed a $\Theta$-edge of $S$.

Corollary 6.2 Suppose that $J$ is an edge of $S$ which is not sharp-angled. Then there exists a $J$-deformation $\delta$ of $S$ such that $\delta(J)$ is sharp-angled and such that $\delta$ is the restriction of an automorphism of $\langle S\rangle$. 
Proof Put $J=\{r, s\}$. By Lemma 5.4, we can find an element $\omega \in\langle J\rangle$ such that $\omega r \omega^{-1}$ and $s$ generate $\omega \in\langle J\rangle$ and such that $\left\{\omega r \omega^{-1}, s\right\}$ is sharp-angled. By the previous proposition, we know that $J$ is a $\Theta$-edge of $S$ and hence, by Proposition 4.6, we can find an $(r, s, \omega)$-deformation of $S$ which extends to an automorphism of $\langle S\rangle$ and we are done.

Conclusion of the proof of Theorem 1 Let $S \subset R^{W}$ be a Coxeter generating set which is not sharp-angled. Suppose $S$ contains $n \geq 1$ edges which are not sharpangled and choose one of them. Call it $J$. By the previous corollary, there exists a $J$-deformation $\delta$ of $S$ which extends to an automorphism of $W$ (because $\langle S\rangle=W$ ) and such that $\delta(J)$ is sharp-angled. Let $J^{\prime}$ be an edge of $S$ different from $J$. Then $\delta\left(J^{\prime}\right)$ is $W$-conjugate to $J^{\prime}$ by Property (AD4) of $\delta$; in particular, $\delta\left(J^{\prime}\right)$ is sharpangled if and only if $J^{\prime}$ is sharp-angled. Hence the number of edges in $\delta(S)$ which are not sharp-angled is $n-1$. Thus the statement follows by an obvious induction on the number of edges of $S$ which are not sharp-angled.

\section{Angle-deformations involving $H_{k}$}

\subsection{Coxeter systems of type $H_{3}$}

Lemma 7.1 Let $(W, S)$ be a Coxeter system of type $H_{3}$, where $S=\{r, s, t\}$ and $o(r s)=5, o(s t)=3$. Set $\omega:=t$ srtst $, \pi:=\operatorname{tr} s$ and define $\delta: S \rightarrow W$ by $\delta(r):=$ $r s r, \delta(s):=s$ and $\delta(t):=\omega t \omega^{-1}$. Then we have the following:

(1) $\omega s \omega^{-1}=s, \omega t \omega^{-1}=\pi r \pi^{-1}, \pi t \pi^{-1}=r s r$.

(2) There is an automorphism $\alpha$ of $W$ which extends $\delta$.

(3) $\delta$ is an $(r, s, s r s)$-deformation of $(W, S)$.

Proof Part (1) is a straightforward calculation. Moreover, it is clear that $\delta(S)$ is contained in $S^{W}$ and that it generates $W$. It follows from (1) that $\{\delta(s), \delta(t)\}=\{s, t\}^{\omega}$ and $\{\delta(r), \delta(t)\}=\{r, t\}^{\pi}$. Furthermore, we have $o(\delta(r) \delta(s))=o($ rs r s $)=5$. By the universal property of Coxeter systems, it follows that $\delta$ extends to an endomorphism $\alpha$ of $W$. Since $\delta(S)$ generates $W, \alpha$ is surjective and hence an automorphism because $W$ is finite. This finishes (2) and shows in particular that $\delta(S)$ is a Coxeter generating set. Assertion (3) is now a consequence of the information collected so far. 
Corollary 7.2 Let $(W, S), \omega, \pi$ and $\delta$ be as in the previous lemma and set $c:=r s r s$, $\omega_{1}:=c \omega, \pi_{1}:=c \pi$ and $\delta_{1}:=\gamma_{c} \circ \delta$, where $\gamma_{c}$ is the inner automorphism $w \mapsto c w c^{-1}$ of $W$. Then we have the following:

(1) $\omega_{1}=$ rstsrst, $\pi_{1}=$ rsrsts.

(2) $\omega_{1} s \omega_{1}^{-1}=s r s, \omega_{1} t \omega_{1}^{-1}=\pi_{1} r \pi_{1}^{-1}$ and $\pi_{1} t \pi_{1}^{-1}=r$.

(3) There is an automorphism $\alpha_{1}$ of $W$ which extends $\delta_{1}$.

(4) $\delta_{1}$ is an $(s, r, r s r)$-deformation of $(W, S)$.

Proof Assertions (1) and (2) are straightforward calculations. Since $\gamma_{c}$ is a reflectionpreserving automorphism of $W$, Assertions (3) and (4) follow from Assertions (2) and (3) of the previous lemma, respectively.

Corollary 7.3 Let $(W, S)$ be a Coxeter system of type $H_{3}$ where $S=\{r, s, t\}$ and $o(r s)=5, o(r t)=3$. Set $\omega:=\operatorname{sistrsrt}, \pi:=\operatorname{sis} s t r$ and define $\delta: S \rightarrow W$ by $\delta(r):=r s r, \delta(s):=s$ and $\delta(t):=\omega t \omega^{-1}$. Then we have the following:

(1) $\omega r \omega^{-1}=r s r, \omega t \omega^{-1}=\pi s \pi^{-1}$ and $\pi t \pi^{-1}=s$.

(2) There is an automorphism $\alpha$ of $W$ which extends $\delta$.

(3) $\delta$ is an $(r, s, s r s)$-deformation of $(W, S)$.

Proof This follows by exchanging the roles of $r$ and $s$ in the previous corollary.

Remark Corollary 7.3 is obtained from Lemma 7.1 by conjugating by $r s r s$ and then relabelling. We refer to this technique again in Section 7.7 without giving further details.

\subsection{Coxeter systems of type $H_{4}$}

Throughout this subsection, $(W, S)$ is a Coxeter system of type $H_{4}$, where $S=$ $\{r, s, t, u\}$ and $o(r s)=5, o(s t)=3$. Set $J:=\{r, s\}, \omega_{1}:=r$ sturstrststusrstrs, $\omega_{2}:=$ tsrsrutsrstsrsutsrsr $\omega_{3}:=$ srsrutsrstsrsutsrstsr $, \omega:=$ rsrst $\omega_{2}$, $\pi:=\omega \omega_{1} u t u, \tau:=\operatorname{trs} \omega_{3} \omega^{-1}$ and define $\delta: S \rightarrow W$ by $\delta(r):=r s r, \delta(s):=s$, $\delta(t):=\omega t \omega^{-1}$ and $\delta(u)=u$.

Lemma 7.4 We have the following:

a) $\pi r \pi^{-1}=r s r, \omega s \omega^{-1}=s, \omega t \omega^{-1}=\pi t \pi^{-1}$ and $\omega u \omega^{-1}=u=\pi u \pi^{-1}$.

b) $\{\delta(r), \delta(t)\}=\{r, t\}^{\pi},\{\delta(r), \delta(u)\}=\{r, u\}^{s r s},\{\delta(s), \delta(t)\}=\{s, t\}^{\omega}$, $\{\delta(s), \delta(u)\}=\{s, u\}^{1_{W}}$ and $\{\delta(t), \delta(u)\}=\{t, u\}^{\omega}$. 
c) $\tau r s r \tau^{-1}=r s r, \tau s \tau^{-1}=s$ and $\tau \omega t \omega^{-1} \tau^{-1}=(\operatorname{tsrtst}) t(\operatorname{tsr} s t)^{-1}$.

Proof The relations in a) and c) are easily deduced from relations given in Franzsen and Howlett [8, page 333], and b) is an immediate consequence of a).

Note The relations for $\tau$ will only be needed in Section 10 .

Proposition 7.5 $\delta$ is an $(r, s, s r s)$-deformation of $S$ which extends to an automorphism of $W$.

Proof Clearly, $r s r=(s r s) r(s r s)$ and $s$ generate $\langle J\rangle$ and $\delta(S)$ generates $W$. Setting $\mathbf{K}:=\{S\}$, it follows that $\delta$ has Properties a), b) and c) of Proposition 3.4, while Property d) is a consequence of the previous lemma. This proves the claim.

\subsection{Conditions (TWa)-(TWt)}

Throughout this subsection, $(W, S)$ is a Coxeter system and $K$ is a subset of $S$ of type $H_{k}$, where $k \in\{3,4\}$ and where $r, s, t \in K$ are such that $o(r s)=5$ and $o(s t)=3$; if $k=4$, the unique element in $K \backslash\{r, s, t\}$ is denoted by $u$. Furthermore, we put $J:=\{r, s\}$ and $\omega:=$ tsrtst if $k=3, \omega:=r \operatorname{srs} \omega_{2}$ if $k=4, \pi:=\operatorname{trs}$ if $k=3$ and $\pi:=\operatorname{rssr} \omega_{2} \omega_{1} u t u$ if $k=4$, where $\omega_{1}$ and $\omega_{2}$ are as in Section 7.2.

Definition 7.6 Let $a \in J$. We say that $K$ is an $a$-special subset of $S$ or that $K$ is $a$-special in $S$ if the following two Conditions (TWa) and (TWt) hold.

(TWa) For all $x \in S \backslash K$ we have $o(x a) \in\{2, \infty\}$, and if $o(x a)=2$ then $x \in J^{\perp}$.

(TWt) If $y \in J^{\perp} \backslash K$ is such that $o(x y)<\infty$ for some $x \in J^{\infty} \cup\{t\}$, then $y \in K^{\perp}$.

Lemma 7.7 Let $a \in J$ be such that $K$ is a-special in $S$. Then we have the following.

a) $\left\{K, J^{\infty}, J^{\perp} \backslash K\right\}$ is a partition of $S$; if $k=3$ then $K \cap J^{\perp}=\varnothing$ and if $k=4$ then $K \cap J^{\perp}=\{u\}$.

b) If $y \in J^{\perp}$ is such that $o(x y)<\infty$ for some $x \in J^{\infty} \cup\{t\}$, then $y$ commutes with $\omega$ and with $\pi$.

Proof Part a) is immediate and Part b) is a consequence of (TWt) and Lemma 7.4 a). 


\subsection{Angle-deformations for $a$-special subsets of $S$}

We adopt the hypotheses of the previous subsection. Furthermore, we assume that $a \in J$ is such that $K$ is $a$-special in $S$.

We define the mapping $\delta: S \rightarrow W$ as follows. We put $\delta(r):=r s r, \delta(y):=y$ for all $y \in\{s\} \cup J^{\perp}$ and $\delta(t):=\omega t \omega^{-1}$. Let $x \in J^{\infty}$. Then we put $\delta(x):=\omega x \omega^{-1}$ if $a=r$ and $\delta(x):=\pi x \pi^{-1}$ if $a=s$.

Lemma 7.8 The mapping $\delta$ has the following properties.

a) $\delta(r)=(s r s) r(s r s)$ and $\delta(s)=s$ generate $\langle J\rangle$.

b) $\delta(S)$ generates $W$.

c) $\left.\delta\right|_{K}$ is an $(r, s, s r s)$-deformation of $K$ which extends to an automorphism of $\langle K\rangle$.

d) For each $x \in S$, there exists an element $w_{x} \in\langle K\rangle$ such that $\delta(x)=w_{x} x w_{x}^{-1}$.

Proof Assertion a) is obvious. Assertions b) and d) are immediate consequences of the definition of $\delta$. Finally, Assertion c) is a consequence of Lemma 7.1 if $k=3$ and of Proposition 7.5 if $k=4$.

Lemma 7.9 Let $E$ be an edge of $S$ different from $J$. If $k=3$ and $a=s$, suppose in addition that $E$ is not of the form $\{z, x\}$ with $z \in\{r, t\}$ and $x \in J^{\infty}$. Then there exists an element $w_{E} \in W$ with $\delta(E)=E^{w_{E}}$.

Proof Let $E=\{x, y\}$ be such an edge of $S$.

If $E$ is contained in $K$, the assertion follows from Lemma 7.1 for $k=3$ and Lemma 7.4 for $k=4$.

If $E$ is contained in $J^{\infty}$, then we may choose $w_{E}=\omega$ if $a=r$ and $w_{E}=\pi$ if $a=s$.

If $E$ is contained in $\{s\} \cup J^{\perp}$, we may choose $w_{E}=1_{W}$.

If $E$ is contained in $\{r\} \cup J^{\perp}$, we may choose $w_{E}=s r s$.

Suppose $E$ is contained in $\{t\} \cup J^{\perp}$. As the case $E \subseteq J^{\perp}$ is already covered by the above, we may assume that $E=\{t, y\}$ for some $y \in J^{\perp}$. Since $o(y t)$ is finite, it follows from Lemma $7.7 \mathrm{~b}$ ) that $y$ commutes with $\omega$. Hence we may choose $w_{E}=\omega$.

Suppose now that $x \in J^{\infty}$ and $y \in J^{\perp}$. Again by Lemma $7.7 \mathrm{~b}$ ), we know that $y$ commutes with $\omega$ and with $\pi$. Hence, we may choose $w_{E}=\omega$ if $a=r$ and $w_{E}=\pi$ if $a=s$. 
Up to renaming the elements of $E$, we are now left with the case where $x \in\{r, s, t\}$ and $y \in J^{\infty}$.

Suppose first that $a=r$. Then the case $x=r$ is not possible and hence $E$ is contained in $\{s, t\} \cup J^{\infty}$. As $s$ commutes with $\omega$ (by Lemma 7.4 a)), we may thus choose $w_{E}=\omega$.

Suppose now that $a=s$. Then the case $x=s$ is not possible and by hypothesis, we only have to consider the case $k=4$. In view of the relations given in Lemma $7.4 \mathrm{~b}$ ), we may choose $w_{E}=\pi$ in this case, and we are done.

Proposition 7.10 If $(a, k) \neq(s, 3)$, then $\delta$ is an $(r, s, s r s)$-deformation of $S$ which is the restriction of an automorphism of $W$.

Proof Setting $\mathbf{K}=\{K\}$ in Proposition 3.4, the two previous lemmas show that $\delta$ has the required properties and we are done.

Lemma 7.11 Suppose $(a, k)=(s, 3)$ and let $x \in J^{\infty}$. Then $\delta(\{r, x\})=\{t, x\}^{\pi}$ and $\delta(\{t, x\})=\{r, x\}^{\pi}$.

Proof This is an immediate consequence of the relations given in Lemma 7.1 and the definition of $\delta$.

\subsection{K-Mirrors}

Throughout this subsection, let $(W, S)$ be a Coxeter system and let $K=\{r, s, t\} \subseteq S$ be of type $\mathrm{H}_{3}$ such that $o(r s)=5$ and $o(s t)=3$.

Definition 7.12 The $K$-mirror of $(W, S)$ is the Coxeter system $(\bar{W}, \bar{S})$ with the property that there exists a bijection $x \mapsto \bar{x}$ from $S$ onto $\bar{S}$ such that $o(\bar{r} \bar{x})=o(t x)$ and $o(\bar{t} \bar{x})=o(r x)$ if $x \in J^{\infty}$, and $o(\bar{x} \bar{y})=o(x y)$ in the remaining cases.

Remark Let $(\bar{W}, \bar{S})$ be the $K$-mirror of $(W, S)$ and for each $X \subseteq S$, put $\bar{X}:=\{\bar{x} \mid$ $x \in X\}$. Then $\bar{K}$ is a subset of $\bar{S}$ of type $H_{3}$ and $(W, S)$ is the $\bar{K}$-mirror of $(\bar{W}, \bar{S})$.

Remark Let $(\bar{W}, \bar{S})$ be the $K$-mirror of $(W, S)$. Then we have an obvious bijection between the edges of $S$ and the edges of $\bar{S}$ which we will call the canonical bijection and which will be denoted by $\theta$.

The following lemma is obvious.

Lemma 7.13 Let $(\bar{W}, \bar{S})$ be the $K$-mirror of $(W, S)$. Then $K$ is $s$-special in $S$ if and only if $\bar{K}$ is $\bar{S}$-special in $\bar{S}$. 


\subsection{The case $(a, k)=(s, 3)$}

Throughout this subsection, let $(W, S)$ be a Coxeter system and let $K=\{r, s, t\} \subseteq S$ be of type $H_{3}$ such that $o(r s)=5$ and $o(s t)=3$. We put $\omega:=t s r t s t, \pi:=t r s$ and $J:=\{r, s\}$. Moreover, $(\bar{W}, \bar{S})$ denotes the $K$-mirror of $(W, S)$. We assume furthermore that $K$ is $s$-special in $S$. Note that this implies that $\bar{K}$ is $\bar{S}$-special in $\bar{S}$. We define the mapping $\delta: S \rightarrow W$ by $\delta(x):=x$ if $x \in J^{\perp} \cup\{s\}, \delta(x):=\pi x \pi^{-1}$ if $x \in J^{\infty}, \delta(r):=r s r$ and $\delta(t):=\omega t \omega^{-1}$.

Lemma 7.14 Let $\{x, y\}$ be an edge of $S$. Then $o(\delta(x) \delta(y))=o(\bar{x} \bar{y})$.

Proof This is a consequence of Lemmas 7.9 and 7.11.

Lemma 7.15 $\delta(S)$ is a Coxeter generating set of $W$. Moreover, there exists a bijection $\Delta$ from the set of edges of $S$ onto the set of edges of $\delta(S)$ such that $\Delta(J)=\{r s r, s\}$ and such that for each edge $E \neq J$ of $S$, there exists $w_{E} \in W$ with $\Delta(E)=E^{w_{E}}$.

Proof By the universal property of $(\bar{W}, \bar{S})$ and Lemma 7.14 and Lemma 7.8, there is an epimorphism $\bar{\beta}: \bar{W} \rightarrow W: \bar{x} \mapsto \delta(x)$ with the following properties:

a) $\left.\bar{\beta}\right|_{\langle\bar{K}\rangle}$ is an isomorphism from $\langle\bar{K}\rangle$ onto $\langle K\rangle$.

b) For each $x \in S$, there exists an element $w_{x} \in\langle K\rangle$ such that $\bar{\beta}(\bar{x})=w_{x} x w_{x}^{-1}$.

By Lemma 7.13, $\bar{K}$ is $\bar{S}$-special in $\bar{S}$. Hence, by defining $\bar{\omega}, \bar{\pi} \in \bar{W}$ and $\bar{\delta}: \bar{S} \rightarrow \bar{W}$ for $(\bar{W}, \bar{S})$, we obtain also an epimorphism $\beta: W \rightarrow \bar{W}$ with the following properties:

a) $\left.\beta\right|_{\langle K\rangle}$ is an isomorphism from $\langle K\rangle$ onto $\langle\bar{K}\rangle$.

b) For each $x \in S$, there exists an element $\bar{w}_{x} \in\langle\bar{K}\rangle$ such that $\beta(x)=\bar{w}_{x} \bar{x} \bar{w}_{x}^{-1}$.

We put $\alpha:=\bar{\beta} \circ \beta$ and for each $x \in S$, we set $v_{x}:=\bar{\beta}\left(\bar{w}_{x}\right) w_{x}$. Then $\alpha: W \rightarrow W$ is an epimorphism with the following properties:

a) $\left.\alpha\right|_{\langle K\rangle}$ is an automorphism of $\langle K\rangle$.

b) For each $x \in S$, we have $v_{x} \in\langle K\rangle$ and $\alpha(x)=v_{x} x v_{x}^{-1}$.

Now, it follows from Proposition 2.3 (with $\mathbf{K}=\{K\}$ ) that $\alpha$ is an automorphism of $W$. In particular, $\bar{\beta}$ is an isomorphism. As $\delta(S)=\bar{\beta}(\bar{S})$, the set $\delta(S)$ is a Coxeter generating set of $W$.

It remains to find an appropriate $\Delta$. As $\bar{\beta}$ is an isomorphism, we have a canonical bijection $\Delta_{1}$ from the set of edges of $\bar{S}$ onto the set of edges of $\delta(S)$. Let $\theta$ be the canonical bijection from the set of edges of $S$ onto the set of edges of $\bar{S}$. It is then readily verified, using Lemmas 7.9 and 7.11, that $\Delta:=\Delta_{1} \circ \theta$ is the required bijection. This finishes the proof of the lemma. 
Proposition 7.16 $\delta$ is an $(r, s, s r s)$-deformation of $(W, S)$.

Proof This is a consequence of the two previous lemmas.

\subsection{The relabeled version}

Throughout this subsection, $(W, S)$ is a Coxeter system and $K$ is a subset of $S$ of type $H_{k}$, where $k \in\{3,4\}$ and where $r, s, t \in K$ are such that $o(r s)=5$ and $o(r t)=3$; if $k=4$, the unique element in $K \backslash\{r, s, t\}$ is denoted by $u$. Define $\bar{\omega}_{i}$ for $i \in\{1,2\}$ by exchanging $\mathrm{r}$ and $\mathrm{s}$ in the expression of $\omega_{i}$ given in Section 7.2, where $t$ and $u$ are as above. Also, let $c:=r s r s$ and $\bar{c}:=s r s r$. We put $J:=\{r, s\}, \omega:=\bar{c}$ trstrt $=$ srstrsrt if $k=3, \omega:=\bar{c} \operatorname{sis} r \bar{\omega}_{2}=r \bar{\omega}_{2}$ if $k=4$, $\pi:=\bar{c} t s r=s r s r s t r$ if $k=3$ and $\pi:=\bar{c} s r s s \bar{\omega}_{2} \bar{\omega}_{1} u t u=r \bar{\omega}_{2} \bar{\omega}_{1} u t u$ if $k=4$. We assume that $a \in J$ is such that $K$ is $a$-special in $S$ and we define $\delta: S \rightarrow W$ as follows. We put $\delta(r):=r s r, \delta(y):=y$ for all $y \in\{s\} \cup J^{\perp}$ and $\delta(t):=\omega t \omega^{-1}$. Let $x \in J^{\infty}$. Then we put $\delta(x):=\omega x \omega^{-1}$ if $a=s$ and $\delta(x):=\pi x \pi^{-1}$ if $a=r$.

The following proposition is obtained from Proposition 7.10 and Proposition 7.16 by relabelling.

Proposition 7.17 The mapping $\delta$ is an $(r, s, s r s)$-deformation of $(W, S)$. Moreover, if $(k, a) \neq(3, r)$, it is the restriction of an automorphism of $W$.

\section{$8 \Delta$-edges}

\subsection{Some particular diagrams}

Throughout this subsection, we put $\lambda:=2 \cos (\pi / 5)$.

Let $\left(W_{1}, R_{1}\right)$ be a Coxeter system whose diagram is as in Figure 2 and let $\left(W_{2}, R_{2}\right)$ be a Coxeter system whose diagram is as in Figure 3. Hence, we have $R_{1}=\{r, s, t\} \cup X$ and $R_{2}=\{r, s, t, u\} \cup X$ where $X=\{S(i) \mid 1 \leq i \leq n\}$.

For $k=1,2$, we consider the geometric representation of $\left(W_{k}, R_{k}\right)$ and its root system $\Phi_{k}$; in particular we identify $W_{k}$ with its image in $O\left(V_{k}, b_{k}\right)$.

We put $\alpha_{1}:=r s\left(e_{r}\right)=\lambda e_{r}+\lambda e_{s}, \Pi_{1}:=\left\{\alpha_{1}, e_{t}\right\} \cup\left\{e_{S(i)} \mid 1 \leq i \leq n\right\}, S_{1}:=\left\{\rho_{\alpha} \mid\right.$ $\left.\alpha \in \Pi_{1}\right\}$ and $\omega_{1}:=r s t$.

We put $\alpha_{2}:=\operatorname{srstrs}\left(e_{r}\right)=(\lambda+1) e_{r}+2 \lambda e_{s}+\lambda e_{t}, \Pi_{2}:=\left\{\alpha_{2}, e_{u}\right\} \cup\left\{e_{S(i)} \mid 1 \leq i \leq n\right\}$, $S_{2}:=\left\{\rho_{\alpha} \mid \alpha \in \Pi_{2}\right\}$ and $\omega_{2}:=$ srstrsut. 


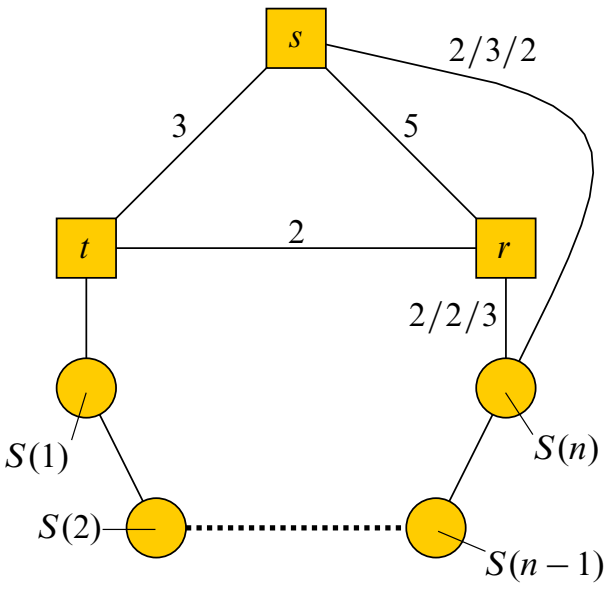

Figure 2. (DE3): $n \geq 2$ and $\overline{X_{1} t}=\infty$ and $X_{n} \subseteq$ $\{r, s\}^{\infty}$.

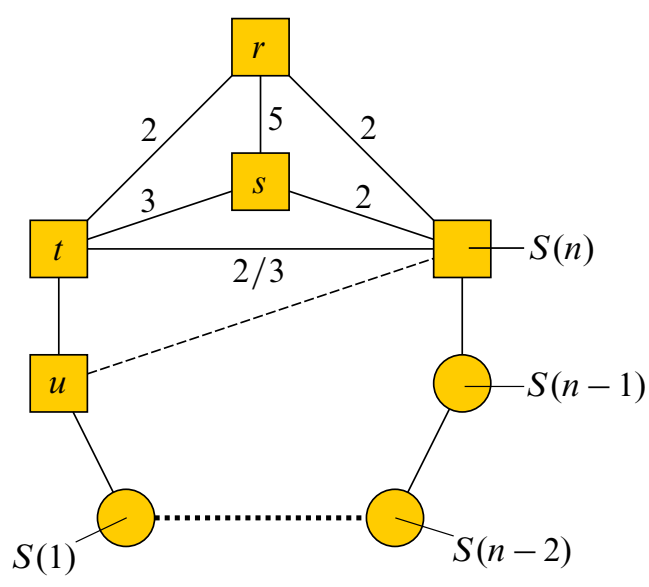

Figure 3. (DE4): $n \geq 2$ and $\overline{X_{1} u}=\infty$ and $X_{n} \subseteq$ $\{r, s, t\}^{\infty}$ and $r, s, t, u$ form an $\mathrm{H}_{4}$.

The following facts are easily verified for $k=1,2$ :

a) $\rho_{\alpha_{k}}=\omega_{k} r \omega_{k}^{-1}$.

b) $\Pi_{k}$ is a root subbase of $\Phi_{k}$; in particular $S_{k}$ is a fundamental set.

c) $\Gamma\left(S_{k}\right)$ is a chordfree circuit.

d) $\omega_{1} s \omega_{1}^{-1}=t$ and $\omega_{2} s \omega_{2}^{-1}=u$.

\subsection{Coxeter systems containing some particular subsystems}

Throughout this subsection, $(W, R)$ is a Coxeter system and $W$ is identified with its image in $O(V, b)$ via its geometric representation.

Proposition 8.1 For $k=1,2$, let $R_{k} \subseteq R^{W}$ be a fundamental set of reflections and put $W_{k}:=\left\langle R_{k}\right\rangle$. Suppose that $\left(W_{k}, R_{k}\right)$ is a Coxeter system whose diagram is as in Figure 2 if $k=1$ and as in Figure 3 if $k=2$. Then $\{r, s\}$ is sharp-angled.

Proof For $k=1,2$, we define $\omega_{k} \in W_{k}$ as in the previous subsection. We put $S_{1}:=\left(R_{1} \backslash\{r, s\}\right) \cup\left\{\omega_{1} r \omega_{1}^{-1}\right\}$ and $S_{2}:=\left(R_{2} \backslash\{r, s, t\}\right) \cup\left\{\omega_{2} r \omega_{2}^{-1}\right\}$. By the considerations above, we know that the set $S_{k}$ is a fundamental set of reflections. Moreover, $\Gamma\left(S_{k}\right)$ is a chordfree circuit. By Theorem 5.9, it follows that $S_{k}$ is sharpangled. Hence $\left\{\omega_{1} r \omega_{1}^{-1}, t\right\}$ and $\left\{\omega_{2} r \omega_{2}^{-1}, u\right\}$ are sharp-angled. As $\omega_{k}$ is an element of $W$ which conjugates $\{r, s\}$ onto $\left\{\omega_{1} r \omega_{1}^{-1}, t\right\}$ for $k=1$, and onto $\left\{\omega_{k} r \omega_{k}^{-1}, u\right\}$ for $k=2$, it follows that $\{r, s\}$ is sharp-angled as well. 
Corollary 8.2 Let $S \subseteq R^{W}$ be a fundamental set of reflections and let $J=\{r, s\}$ be an edge of $S$ such that $o(r s)=5$, and which is not sharp-angled. Then there is no subset $K$ of $S$ as in Figure 2 or Figure 3.

\subsection{Definition of $\Delta$-edges}

Definition 8.3 Let $W$ be a group and $S$ a subset of involutions of $W$. Let $J=\{r, s\}$ be an edge of $S$. We call $J$ a $\Delta-e d g e$ of $S$ if there is no subset $K$ of $S$ containing $J$ having one of the following properties:

(DE1) $\Gamma(K)$ is nonspherical, 2-spherical and irreducible.

(DE2) $\Gamma(K)$ is a chordfree circuit of length at least 4.

(DE3) $\Gamma(K)$ is a diagram as shown in Figure 2.

(DE4) $\Gamma(K)$ is a diagram as shown in Figure 3.

Remark Note that if $o(r s) \neq 5$, then $J$ is a $\Delta$-edge if and only if (DE1) and (DE2) hold; if $o(r s)=5$, the same remains true if there is no subset of type $\mathrm{H}_{3}$ containing $\mathrm{J}$.

The definition of $\Delta$-edges is motivated by the following proposition, which is a consequence of Theorem 5.9 and Corollary 8.2.

Proposition 8.4 Let $(W, R)$ be a Coxeter system, let $S \subseteq R^{W}$ be a fundamental set of reflections and suppose that $J$ is an edge of $S$ which is not sharp-angled with respect to $R$. Then $J$ is a $\Delta$-edge of $S$.

\section{$9 \Delta$-edges of type $\mathrm{H}_{2}$}

Throughout this section, $(W, S)$ is a Coxeter system and $J=\{r, s\} \subseteq S$ is a $\Delta$-edge of $(W, S)$ with $o(r s)=5$. Moreover, we define several subsets of $S$ as follows.

- $T:=\left\{t \in S \mid \operatorname{type}(\{r, s, t\})=H_{3}\right\}=T_{r} \amalg T_{s}$, where $T_{r}:=\left\{t \in T \mid m_{r t}=3\right\}$ and $T_{s}:=\left\{t \in T \mid m_{s t}=3\right\}$.

- For a $J$-component $L$, put $T_{L}:=\left\{t \in T \mid \exists x \in L: m_{x t}<\infty\right\}$.

- $U:=\left\{u \in S \mid \exists t \in T\right.$ such that type $\left.(\{r, s, t, u\})=H_{4}\right\}$.

- For $t \in T$, set $U_{t}:=\left\{u \in U \mid \operatorname{type}(\{r, s, t, u\})=H_{4}\right\}$.

- For $t \in T$ and $L$ a $J_{t}$-component, let $U_{L}:=\left\{u \in U_{t} \mid \exists x \in L: m_{x u}<\infty\right\}$.

- For $t \in T$ and $u \in U_{t}$, let $J_{t}:=J \cup\{t\}$ and $J_{t, u}:=J \cup\{t\} \cup\{u\}$.

- For $u \in U$, set $T_{u}:=\left\{t \in T \mid \operatorname{type}(\{r, s, t, u\})=H_{4}\right\}$.

- $T^{3}:=\left\{t \in T \mid U_{t}=\varnothing\right\}$.

- $T^{4}:=T \backslash T^{3}$.

- For $a \in J$ and $k \in\{3,4\}$, put $T_{a}^{k}:=T_{a} \cap T^{k}$. 


\subsection{Some preliminary observations}

Lemma 9.1 $J$ is flexible.

Proof This is Lemma 2.8.

Lemma 9.2 There are no edges in $T$ and for each $t \in T$, there are no edges in $U_{t}$.

Proof This follows from (DE1).

\subsection{Flexibility of $J_{t}$ and consequences}

Proposition 9.3 For all $t \in T$, the set $J_{t}$ is flexible.

Proof Let $t \in T$ and let $L$ be a $J_{t}$-component. If $L$ is also a $J$-component, then $L$ is flexible by Lemma 9.1 and we are done. So, we may assume there exists an $x \in L$ such that $x \in J^{\text {fin }}$ (thus $m_{x t}=\infty$ ). Suppose by contradiction there exists $y \in L$ such that $m_{y t}<\infty$. Then $m_{y r}=\infty$ or $m_{y s}=\infty$.

Let $x=x_{0}, x_{1}, \ldots, x_{k}=y$ be a minimal path in $L$ joining $x$ to $y$. Define

and

$$
M:=\min \left\{i \mid 0<i \leq k ; m_{x_{i} t}<\infty\right\}
$$

$$
m:=\max \left\{i \mid 0 \leq i<M ; x_{i} \in J^{\mathrm{fin}}\right\} \text {. }
$$

Then the subpath $x_{m}, x_{m+1}, \ldots, x_{M}$ from $x_{m}$ to $x_{M}$ is still minimal, hence chordfree, and possesses the following properties:

(1) $\left(m_{x_{m} r}, m_{x_{m} s}\right) \in\{(2,2),(2,3),(3,2)\}$ (by (DE1)).

(2) $m_{x_{i} t}=\infty$ for all $i$ such that $m \leq i<M$ (by definition of M).

(3) $\quad x_{i} \in J^{\infty}$ for all $i$ such that $m<i \leq M$ (by definition of $\mathrm{m}$ ).

Moreover, $m_{x_{M} t}<\infty$. Thus, we obtain a subgraph $\left\{r, x_{m}, x_{m+1}, \ldots, x_{M}, t, s\right\}$ as pictured in Figure 4, contradicting (DE3).

Corollary 9.4 Let $t \in T$ and let $L$ be a $J$-component such that there exists $z \in L$ with $o(z t)<\infty$. If $y \in J^{\text {fin }} \backslash\{t\}$ is such that there exists an $x \in L$ with $o(x y)<\infty$, then $y \in J_{t}^{\text {fin }}$.

Proof Let $L^{\prime}$ be the $J_{t}$-component containing $L$. If $o(y t)=\infty$, we get $y \in L^{\prime}$ because $o(x y)<\infty$. But then $z$ and $y$ belong to $L^{\prime}$, contradicting the fact that $J_{t}$ is flexible. Hence $o(y t)<\infty$ and so $y \in J_{t}^{\text {fin }}$ because $y \in J^{\text {fin }}$ by assumption. 


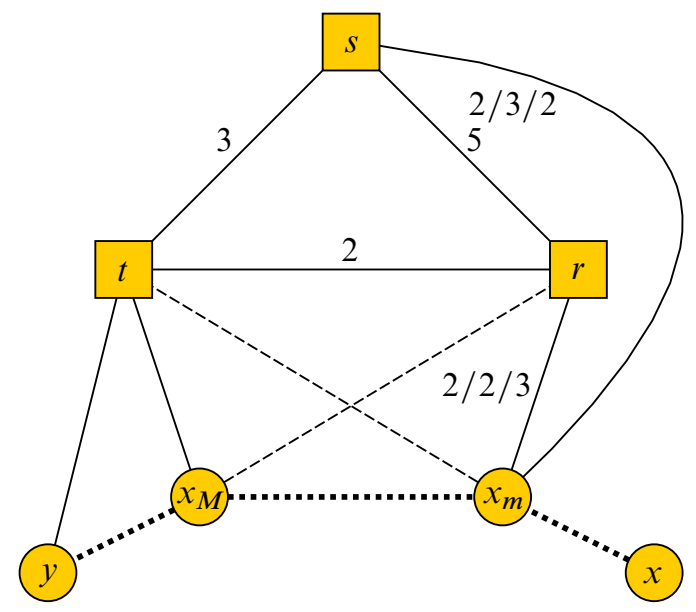

Figure 4. Contradicts (DE3)

Corollary 9.5 Let $L$ be a $J$-component, then $\left|T_{L}\right| \leq 1$.

Proof This follows from the previous corollary and Lemma 9.2.

Definition 9.6 Let $L$ be a $J$-component. If $T_{L}$ is nonempty, then $t(L)$ denotes its unique element; if $T_{L}$ is empty, we put $t(L):=\infty$.

\subsection{Flexibility of $J_{t, u}$ and consequences}

Proposition 9.7 Let $t \in T$ and $u \in U_{t}$. Then $J_{t, u}$ is flexible.

Proof Let $L$ be a $J_{t, u}$-component. If $L$ is also a $J_{t}$-component, then it is free by Proposition 9.3 and we are done. So, we may assume there exists an $x \in L$ such that $x \in J_{t}^{\text {fin }}$ (thus $m_{x u}=\infty$ ). Suppose by contradiction there exists $y \in L$ such that $m_{y u}<\infty$. Then $y \in J_{t}^{\infty}$.

Let $x=x_{0}, x_{1}, \ldots, x_{k}=y$ be a minimal path in $L$ joining $x$ to $y$. Define

and

$$
\begin{aligned}
M & :=\min \left\{i \mid 0<i \leq k ; m_{x_{i} u}<\infty\right\} \\
m & :=\max \left\{i \mid 0 \leq i<M ; x_{i} \in J_{t}^{\mathrm{fin}}\right\} .
\end{aligned}
$$

Then the subpath $x_{m}, x_{m+1}, \ldots, x_{M}$ from $x_{m}$ to $x_{M}$ is still minimal, hence chordfree, and possesses the following properties:

(1) $\left(m_{x_{m} r}, m_{x_{m} s}, m_{x_{m} t}\right) \in\{(2,2,2),(2,2,3)\}$ (by (DE1)). 
(2) $m_{x_{i} u}=\infty$ for all $i$ such that $m \leq i<M$ (by definition of $\mathrm{M}$ ).

(3) $\quad x_{i} \in J_{t}^{\infty}$ for all $i$ such that $m<i \leq M$ (by definition of $\mathrm{m}$ ).

Moreover, $m_{x_{M} u}<\infty$. Thus, we obtain a subgraph $\left\{r, x_{m}, x_{m+1}, \ldots, x_{M}, u, t, s\right\}$ as pictured in Figure 5, contradicting (DE4).

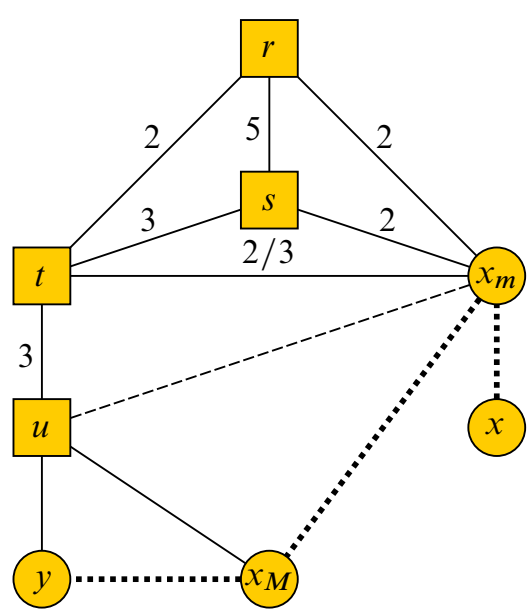

Figure 5. Contradicts (DE4)

Corollary 9.8 Let $t \in T, u \in U_{t}$ and $L$ be a $J_{t}$-component containing an element $z$ with $o(z u)<\infty$. Suppose that $y \in J_{t}^{\text {fin }}$ is such that there exists $x \in L$ with $o(x y)<\infty$. Then $y \in J_{t, u}^{\mathrm{fin}} \cup\{u\}$; in particular, if $y \neq u$, then $y \in J_{t, u}^{\perp}$.

Proof Let $L^{\prime}$ be the $J_{t, u}$-component containing $L$ and suppose $y \neq u$. If $o(y u)=\infty$, we get $y \in L^{\prime}$ since $o(x y)<\infty$. But then $z$ and $y$ belong to $L^{\prime}$, contradicting the flexibility of $J_{t, u}$. Hence $o(y u)<\infty$ and so $y \in J_{t, u}^{\text {fin }}$ because $y \in J_{t}^{\text {fin }}$ by assumption. Now, (DE1) implies that $J_{t, u}^{\mathrm{fin}}=J_{t, u}^{\perp}$, so we are done.

Corollary 9.9 Let $t \in T$ and let $L$ be a $J_{t}$-component. Then $\left|U_{L}\right| \leq 1$.

Proof This follows from the previous corollary.

Definition 9.10 Let $t \in T$ and let $L$ be a $J_{t}$-component. If $U_{L}$ is nonempty, then $u(L)$ denotes its unique element; if $U_{L}$ is empty, we put $u(L):=\infty$. 
Remark Let $t \neq t^{\prime} \in T$. By Lemma 9.2, we can talk about the " $J_{t}$-component containing $t^{\prime \prime}$ " as we will do in the following proposition.

Proposition 9.11 Let $t \neq t^{\prime} \in T$, let $L$ be the $J_{t}$-component containing $t^{\prime}$ and put $K:=J_{t} \cup U_{L}$. Then $J_{t^{\prime}}^{\mathrm{fin}}$ is contained in $K^{\mathrm{fin}} \cup L \cup U_{L}$.

Proof Let $y \in J_{t^{\prime}}^{\mathrm{fin}}$. Then we have in particular $o\left(y t^{\prime}\right)<\infty$. Hence, if $o(y t)=\infty$, we have $y \in L$. Thus we are left with the case where $o(y t)<\infty$. As $y \in J_{t^{\prime}}^{\text {fin }}$, we get that $y \in J_{t}^{\text {fin }}$. In particular, we are already done if $u(L)=\infty$.

Let us now assume that $U_{L} \neq \varnothing$ and put $u:=u(L)$. Then there exists an element $z \in L$ such that $o(u z)<\infty$ and there exists an element $x \in L$ (namely $t^{\prime}$ ) such that $o(x y)<\infty$. As $y \in J_{t}^{\text {fin }}$, the claim follows from Corollary 9.8 .

\subsection{Tameness}

Definition 9.12 Let $t \in T$ and let $K$ be a subset of $S$ containing $J_{t}$. Then $t$ is called tame in $K$ if there is no subset $K^{\prime}$ of $K$ containing $J_{t}$ such that $\Gamma\left(K^{\prime}\right)$ is as in Figure 6. We call $t$ tame, if it is tame in $S$. Otherwise, we call it wild.

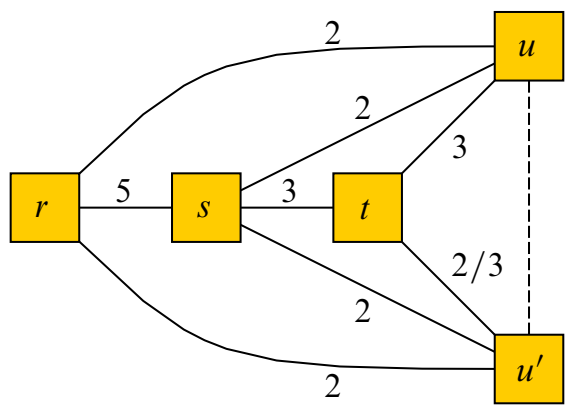

Figure 6. Tameness

Here are some basic observations. The first two of them are obvious whereas the third one is a consequence of Lemma 9.2.

Lemma 9.13 Let $t \in T$ and $K_{1} \subseteq K$ be subsets of $S$ containing $J_{t}$. If $t$ is tame in $K$, then it is tame in $K_{1}$.

Lemma 9.14 If $t \in T^{3}$, then $t$ is tame. 
Lemma 9.15 If $t$ is tame, then $\left|U_{t}\right| \leq 1$.

Let $t \in T$ be tame. Then we put $K_{t}:=J_{t} \cup U_{t}$.

Lemma 9.16 Let $t \in T$ be tame. Then $J_{t}^{\perp}=K_{t}^{\perp}$ and $J_{t}^{\text {fin }} \cup J_{t}=K_{t} \cup K_{t}^{\perp}$.

Proof We start with the first equality which is trivial if $U_{t}$ is empty. Suppose $U_{t}$ is nonempty and let $u$ denote its unique element. Obviously, we have $K_{t}^{\perp} \subset J_{t}^{\perp}$. Let now $y \in J_{t}^{\perp}$. If $o(y u)=\infty$, we get a contradiction to the tameness of $t$ (using (DE1)) and if $2<o(y u)<\infty$, we get a contradiction to (DE1). Hence $o(u y)=2$ and the first equality holds.

The second equality follows now from the fact that $J_{t}^{\text {fin }}=J_{t}^{\perp} \cup U_{t}$ (because of (DE1)), the definition of $K_{t}$ and the first equality.

Lemma 9.17 Let $t \in T$ be tame, $K:=K_{t}$, let $L$ be a $J$-component with $t=t(L)$ and let $a \in J$ be $L$-free. Then $K$ is an $a$-special subset of $S^{\prime}:=K \cup L \cup J^{\perp}$.

Proof Note first that $S^{\prime} \backslash K \subseteq L \cup J^{\perp}$. Thus, as $a$ is $L$-free, Condition (TWa) is obviously satisfied.

We now show that Condition (TWt) holds as well. Note first that $J^{\infty} \cap S^{\prime}=L$. Let $y \in J^{\perp} \backslash K$ such that $o(y x)<\infty$ for some $x \in\left(J^{\infty} \cap S^{\prime}\right) \cup\{t\}=L \cup\{t\}$. We first show that $y \in J_{t}^{\text {fin }}$, which is obvious if $x=t$. Hence we may assume $x \in L$. As $t=t(L)$, there exists $z \in L$ such that $o(t z)<\infty$. Therefore, $y \in J^{\perp} \subseteq J^{\text {fin }} \backslash\{t\}$ and we can apply Corollary 9.4 to see that $y \in J_{t}^{\mathrm{fin}}$.

Now, as $t$ is tame and $y$ is not in $K$, we have $y \in J_{t}^{\perp}$ and we are done if $U_{t}=\varnothing$. Suppose $U_{t} \neq \varnothing$ and let $u$ be the unique element of $U_{t}$. If $o(y u)=\infty$, we get a contradiction to the tameness of $t$ and if $2<o(y u)<\infty$, we get a contradiction to (DE1). Hence $o(y u)=2$ and $y \in K^{\perp}$ because $K=J_{t} \cup\{u\}$ and $y \in J_{t}^{\perp}$.

\subsection{The degree of a subset containing $J$}

Definition 9.18 Let $K$ be a subset of $S$ containing $J$. The degree of $K$ is the number of elements in $K \cap T$ which are wild in $K$. It is denoted by $\operatorname{deg}(K)$.

Here is a preliminary observation.

Lemma 9.19 Let $J \subseteq K_{1} \subseteq K \subseteq S$. Then $\operatorname{deg}\left(K_{1}\right) \leq \operatorname{deg}(K)$. 
Definition 9.20 Let $t \in T$. For each $u \in \widehat{U}_{t}:=U_{t} \cup\{\infty\}$, we define the sets $V_{u}, W_{u}, X_{u}, Y_{u}$ and $Z_{u}$ as follows.

- $V_{\infty}:=J_{t}$ and $V_{u}:=J_{t, u}$ for $u \in U_{t}$.

- $W_{u}:=V_{u} \cup V_{u}^{\perp}$.

- $X_{u}$ is the union of all $J_{t}$-components $L$ such that $u(L)=u$.

- $Y_{u}:=W_{u} \cup X_{u}$.

- $Z_{u}:=Y_{u} \cup Y_{\infty}$.

Lemma 9.21 Let $t \in T$ and $u \in \widehat{U}_{t}$. Then $t$ is tame in $Y_{u}$. In particular, if $t$ is wild then $\operatorname{deg}\left(Y_{u}\right)<\operatorname{deg}(S)$.

The following is a consequence of Proposition 9.11.

Lemma 9.22 Let $t \neq t^{\prime} \in T$ and $u \in \widehat{U}_{t}$. If $t^{\prime}$ is contained in $X_{u}$, then $J_{t^{\prime}}^{\mathrm{fin}} \subseteq Y_{u}$.

Lemma 9.23 Let $u \in U_{t}$. Then $Y_{u} \cap Y_{\infty}=J_{t} \cup J_{t, u}^{\perp}$ and if $E$ is an edge of $Z_{u}$, then $E \subseteq Y_{u}$ or $E \subseteq Y_{\infty}$.

Proof The first statement follows from the definition of the sets $Y_{u}$ and $Y_{\infty}$.

Let $E=\{x, y\}$ be an edge of $Z_{u}$ and suppose that $x \in Y_{u}$ and $y \in Y_{\infty}$.

Suppose first that $x \in X_{u}$. Then $y$ cannot be in $X_{\infty}$ since in that case $x$ and $y$ would be in different $J_{t}$-components. Hence, $y \in J_{t} \cup J_{t}^{\perp}$. If $y \in J_{t} \cup J_{t, u}^{\perp}$, then $y$ is in $Y_{u}$ and we are done. Suppose by contradiction that $y \in J_{t}^{\perp} \backslash J_{t, u}^{\perp}$. Then we have $o(y u)=\infty$ by (DE1). Let $L$ be the $J_{t}$-component containing $x$. Then there is an element $z$ in $L$ such that $o(u z)$ is finite. Let $L^{\prime}$ be the $J_{t, u}$-component containing $L$. Then $x, y$ and $z$ are contained in $L^{\prime}$, contradicting the flexibility of $J_{t, u}$.

Thus we may assume that $x \in J_{t, u} \cup J_{t, u}^{\perp}$. If $x \neq u$, we have $x \in Y_{\infty}$ and we are done. Suppose that $x=u$. Then the case $y \in X_{\infty}$ is not possible, because otherwise we would have $u=u(L)$ for the $J_{t}$-component $L$ containing $y$. Thus we may assume that $y \in J_{t} \cup J_{t}^{\perp}$. By (DE1), we then get $y \in J_{t} \cup J_{t, u}^{\perp}$ and hence $E \subseteq Y_{u}$ in this case.

\section{Existence of angle-deformations}

Throughout this section, $(W, S)$ is a Coxeter system and $J=\{r, s\} \subseteq S$ is a $\Delta$-edge of $(W, S)$ with $o(r s)=5$.

We adopt the notation of the previous section. 


\subsection{Conventions for tame elements and standard deformations}

If $t \in T$ is tame, we fix the following notation:

- By Lemma 9.15, there exists precisely one element in $U_{t}$ for each $t \in T^{4}$, which we will denote by $u_{t}$.

- If $t \in T_{s}^{3}$, we put $\omega_{t}:=t s r t s t$ and $\pi_{t}:=t r s$.

- If $t \in T_{r}^{3}$, we put $\omega_{t}:=$ srstrsrt and $\pi_{t}:=$ srsistr.

- If $t \in T_{s}^{4}$, we put $\omega_{t}:=\operatorname{rsrr} \omega_{2}$ and $\pi_{t}:=\operatorname{rssr} \omega_{2} \omega_{1} t u t$, where $u:=u_{t}$ and $\omega_{1}, \omega_{2}$ are as in Section 7.2.

- If $t \in T_{r}^{4}$, we put $\omega_{t}:=r \bar{\omega}_{2}$ and $\pi_{t}:=r \bar{\omega}_{2} \bar{\omega}_{1} u t u$, where $u:=u_{t}$ and $\bar{\omega}_{1}, \bar{\omega}_{2}$ are as in Section 7.7.

- For $t \in T^{3}$, we put $K_{t}:=J_{t}$ and for $t \in T^{4}$, we put $K_{t}:=J_{t} \cup\left\{u_{t}\right\}$.

- We put $\widehat{T}:=T \cup\{\infty\}, K_{\infty}=J_{\infty}:=J, \omega_{\infty}:=1_{W}$ and $\pi_{\infty}:=\operatorname{srs}$.

- Finally, for $t \in \widehat{T}$, we put $K_{t}^{\mathrm{def}}=K_{t} \cup K_{t}^{\perp}$.

Let $t \in \widehat{T}$ and if $t \neq \infty$, suppose it is tame. We define $\delta_{t}: K_{t}^{\text {def }} \rightarrow\left\langle K_{t}^{\text {def }}\right\rangle$ by $\delta_{t}(r)=r s r, \delta_{t}(s)=s, \delta_{t}(t)=\omega_{t} t \omega_{t}^{-1}$ (for $\left.t \neq \infty\right), \delta_{t}\left(u_{t}\right):=u_{t}$ for $t \in T^{4}$ and $\delta_{t}(x):=x$ for all $x \in K_{t}^{\perp}$.

Proposition 10.1 $\delta_{t}$ is an $(r, s, s r s)$-deformation of $K_{t}^{\mathrm{def}}$.

Proof This is a consequence of Lemma 7.1, Corollary 7.3 and Proposition 7.5 together with its relabeled version.

Definition 10.2 We call $\delta_{t}$ the standard deformation of $K_{t}^{\mathrm{def}}$.

\subsection{Tame angle-deformations}

Definition 10.3 Let $K$ be a subset of $S$ containing $J$ and let $\delta: K \rightarrow\langle K\rangle$ be an $(r, s, s r s)$-deformation of $K$. Then we call $\delta$ tame if for each $t \in T \cap K$ which is tame in $K$, there exists an element $w_{t} \in\langle K\rangle$ such that $\delta(x)=w_{t} \delta_{t}(x) w_{t}^{-1}$ for all $x \in K_{t}^{\mathrm{def}}$.

The goal of this section is to prove the following result.

Theorem 10.4 There exists a tame $(r, s, s r s)$-deformation of $S$. 


\subsection{The tame case}

Throughout this subsection, we assume the following.

(TAME) All elements in $T$ are tame.

For each $t \in \widehat{T}$, let $\delta_{t}: K_{t}^{\text {def }} \rightarrow\left\langle K_{t}^{\text {def }}\right\rangle$ be the standard deformation.

We put $\widehat{J}:=J \cup T \cup J^{\perp}$ and we define $\hat{\delta}: \widehat{J} \rightarrow\langle\widehat{J}\rangle$ by $\left.\widehat{\delta}\right|_{K_{t}^{\text {def }}}:=\delta_{t}$ for each $t \in \widehat{T}$ and $\left.\hat{\delta}\right|_{J \perp}:=\mathrm{id}_{J \perp}$. Note that $\hat{\delta}$ is well-defined.

Our first goal is to prove the following proposition.

Proposition 10.5 $\hat{\delta}$ is a tame $(r, s, s r s)$-deformation of $(\langle\widehat{J}\rangle, \widehat{J})$.

Lemma 10.6 Let $t \in T$ and $y \in J^{\perp}$ such that $o(t y)<\infty$. Then, $y$ commutes with $\omega_{t}$ and $\pi_{t}$.

Proof If $t \in T^{3}$ or if $y \neq u_{t}$, then the tameness of $t$ and (DE1) imply that $y \in J_{t}^{\perp}$, and hence $y \in K_{t}^{\perp}$ by Lemma 9.16 and we are done. If $y=u_{t}$, then the result follows from Lemma 7.4 a).

Lemma 10.7 Let $E:=\{x, y\}$ be an edge of $\widehat{J}$ different from $J$. Then, there is an element $w_{E} \in \bigcup_{t \in \widehat{T}}\left\langle J_{t}\right\rangle$ such that $\hat{\delta}(E)=E^{w_{E}}$.

Proof If $E$ is contained in $J_{t}$ for some $t \in T$, then there exists an element $w_{E} \in\left\langle J_{t}\right\rangle$ such that $\hat{\delta}(E)=E^{w_{E}}$. This follows from Lemma 7.1 and Corollary 7.3.

If $E$ is contained in $J^{\perp} \cup\{s\}$, then $\widehat{\delta}(E)=E^{1_{W}}$. Hence, the case $s \in E$ is settled completely.

Suppose now $x=r$. In this case, we may assume $y \in J^{\perp}$ because the case $y \in T$ is already covered above. For all $y \in J^{\perp}$, we have ysrs $=s r s y$ and therefore $\widehat{\delta}(E)=E^{s r s}$.

By Lemma 9.2, it remains to consider the case where $x \in T$ and $y \in J^{\perp}$. Set $x=t$. As $\{x, y\}$ is an edge, it follows from Lemma 10.6 that $y$ commutes with $\omega_{t}$. Hence we have $\hat{\delta}(E)=E^{\omega_{t}}$ in this case.

Proof of Proposition 10.5 It is readily verified that $\hat{\delta}(\hat{J})$ generates $\langle\widehat{J}\rangle$ and by Lemma 10.7 and Proposition 2.3 (with $\mathbf{K}=\left\{J_{t} \mid t \in \widehat{T}\right\}$ ), it follows that $\hat{\delta}$ extends to an automorphism $\hat{\alpha}$ of $\langle\widehat{J}\rangle$, which implies in particular that $\hat{\delta}(\hat{J})$ is a Coxeter generating set of $\langle\hat{J}\rangle$. Using Lemma 10.7, it is now straightforward to check that $\hat{\delta}$ satisfies Properties (AD1)-(AD4). The tameness of $\hat{\delta}$ is a consequence of its definition. This concludes the proof of Proposition 10.5. 
Let $L$ be a $J$-component and define the set $T_{L} \subseteq T$ as before. Since we assume that $J$ is a $\Delta$-edge, we know by Corollary 9.5 that $\left|T_{L}\right| \leq 1$. We define $t(L)$ as in Section 9.2. Moreover, we put $J_{L}:=K_{t(L)}, K_{L}:=J_{L} \cup J^{\perp} \cup L$ and $M_{L}:=K_{L} \cup T$.

Let $\Pi(L)$ be the set of $L$-free vertices of $J$; since $J$ is flexible (by Lemma 9.1), we know that $\Pi(L) \neq \varnothing$.

For each $J$-component $L$, we define $\gamma_{L} \in\left\langle J_{L}\right\rangle$ as follows.

If $t(L)=\infty$ and $r \in \Pi(L)$, we put $\gamma_{L}:=1_{W}$.

If $t(L)=\infty$ and $\Pi(L)=\{s\}$, we put $\gamma_{L}:=s r s$.

If $t(L) \in T_{s}$ and $r \in \Pi(L)$, we put $a_{L}:=r$ and $\gamma_{L}:=\omega_{t}$.

If $t(L) \in T_{s}$ and $\Pi(L)=\{s\}$, we put $a_{L}:=s$ and $\gamma_{L}:=\pi_{t}$.

If $t(L) \in T_{r}$ and $s \in \Pi(L)$, we put $a_{L}:=s$ and $\gamma_{L}:=\omega_{t}$.

If $t(L) \in T_{r}$ and $\Pi(L)=\{r\}$, we put $a_{L}:=r$ and $\gamma_{L}:=\pi_{t}$.

Finally, we define $\delta_{L}: K_{L} \rightarrow\left\langle K_{L}\right\rangle$ by $\left.\delta_{L}\right|_{J_{L}}:=\left.\delta_{t(L)}\right|_{J_{L}},\left.\delta_{L}\right|_{J \perp}=\mathrm{id}_{J \perp}$ and $\delta_{L}(x):=$ $\gamma_{L} x \gamma_{L}^{-1}$ for all $x \in L$. Note that $\delta_{L}$ is well-defined.

Lemma 10.8 Let $L$ be a $J$-component with $t:=t(L) \neq \infty$. Then $K_{t}$ is an $a_{L^{-}}$ special subset of $K_{L}$.

Proof This is a consequence of Lemma 9.17.

Lemma 10.9 Let $L$ be a $J$-component. Then $\delta_{L}$ is an $(r, s, s r s)$-deformation of $K_{L}$.

Proof This is a consequence of the previous lemma and Propositions 7.10, 7.16 and 7.17 applied to the Coxeter system $\left(\left\langle K_{L}\right\rangle, K_{L}\right)$ if $t(L) \neq \infty$, and of Proposition 4.4 applied to the same Coxeter system otherwise.

Proposition 10.10 Let $L$ be a $J$-component. Define $\widehat{\delta}_{L}: M_{L} \rightarrow\left\langle M_{L}\right\rangle$ by $\left.\widehat{\delta}_{L}\right|_{K_{L}}:=$ $\delta_{L}$ and $\left.\hat{\delta}_{L}\right|_{\hat{J}}:=\hat{\delta}$. Then $\hat{\delta}_{L}$ is an $(r, s, s r s)$-deformation of $M_{L}$.

Proof Note first that $\hat{\delta}_{L}$ is well-defined. By the previous lemma, $\delta_{L}$ is an $(r, s, s r s)-$ deformation of $K_{L}$ and by Proposition $10.5, \hat{\delta}$ is an $(r, s, s r s)$-deformation of $\hat{J}$. As $K_{L} \backslash \widehat{J}=L$ and $\widehat{J} \backslash K_{L}=T \backslash T_{L}$, all edges of $M_{L}$ are contained in at least one of the two sets. Now, as $\delta$ restricted to $M_{L} \cap K_{L}=J_{L} \cup J^{\perp}$ is an $(r, s, r s r)$-deformation of $M_{L} \cap K_{L}$, Proposition 3.3 finishes the proof. 
Theorem 10.11 Let $\delta: S \rightarrow W$ be the mapping defined by $\left.\delta\right|_{M_{L}}:=\widehat{\delta}_{L}$ for each $J$-component $L$. Then $\delta$ is a tame $(r, s, s r s)$-deformation of $(W, S)$.

Proof Note that for two different $J$-components $L$ and $L^{\prime}$, we have $M_{L} \cap M_{L^{\prime}}=$ $T \cup J^{\perp} \cup J$, which is independent of $L$ and $L^{\prime}$. Moreover, $\delta$ restricted to $T \cup J^{\perp} \cup J$ is an $(r, s, r s r)$-deformation of $T \cup J^{\perp} \cup J$. The claim now follows by induction on the number of $J$-components using Propositions 3.3 and 10.10, the tameness being a consequence of Proposition 10.5.

\subsection{Proof of Theorem 10.4}

The theorem will be proved by induction on $\operatorname{deg}(S)$. If $\operatorname{deg}(S)=0$, all elements in $T$ are tame and we are done by Theorem 10.11. Suppose now that the degree of $S$ is at least 1 . Then there exists a wild $t \in T$, which we fix throughout this subsection.

For each $u \in \widehat{U}_{t}$, we define the sets $V_{u}, W_{u}, X_{u}, Y_{u}$ and $Z_{u}$ as in Section 9.5. For $u \in U_{t}$, we put $\tau_{u}:=\tau$ where $\tau$ is defined as in Section 7.2 and $\tau_{\infty}:=1_{W}$.

Let $u \in \widehat{U}_{t}$. By Lemma 9.21, we know that $\operatorname{deg}\left(Y_{u}\right)<\operatorname{deg}(S)$. Thus, we know by induction that there is a tame $(r, s, s r s)$-deformation $\theta_{u}$ of $Y_{u}$. Again by Lemma 9.21, $t$ is tame in $Y_{u}$ and if we define $K_{t}^{\mathrm{def}}$ as in Section 10.1 with respect to $Y_{u}$, we have $W_{u}=K_{t}^{\mathrm{def}}$. Hence, there is an $w_{u} \in\left\langle Y_{u}\right\rangle$ such that $\left.\operatorname{Int}\left(w_{u}\right) \circ \theta_{u}\right|_{W_{u}}$ is the standard deformation of $W_{u}$. We put $\Theta_{u}:=\operatorname{Int}\left(w_{u}\right) \circ \theta_{u}$. The discussion above yields the following.

Lemma 10.12 For each $u \in \widehat{U}_{t}$, there exists a tame $(r, s, s r s)$ deformation $\Theta_{u}$ of $Y_{u}$ such that $\left.\Theta_{u}\right|_{W_{u}}$ is the standard deformation of $W_{u}$.

For each $u \in \widehat{U}_{t}$, let $\Theta_{u}$ be as in the previous lemma and put $\delta_{u}:=\operatorname{Int}\left(\tau_{u}\right) \circ \Theta_{u}$.

Lemma 10.13 For each $u \in \widehat{U}_{t}$ the mapping $\delta_{u}: Y_{u} \rightarrow\left\langle Y_{u}\right\rangle$ is a tame $(r, s, s r s)-$ deformation of $Y_{u}$. Moreover, we have $\left.\delta_{u}\right|_{Y_{u} \cap Y_{\infty}}=\left.\delta_{\infty}\right|_{Y_{u} \cap Y_{\infty}}$. In particular, there exists an $(r, s, s r s)$-deformation $\hat{\delta}_{u}$ of $Z_{u}$ such that $\left.\hat{\delta}_{u}\right|_{Y_{u}}=\delta_{u}$ and $\left.\widehat{\delta}_{u}\right|_{Y_{\infty}}=\delta_{\infty}$.

Proof The first assertion of the lemma is clear, because $\Theta_{u}$ is tame and $\tau_{u} \in\left\langle Y_{u}\right\rangle$.

The second assertion is trivial for $u=\infty$, so we may assume $u \in U_{t}$. First remark that $Y_{u} \cap Y_{\infty}=J_{t} \cup J_{t, u}^{\perp}$ by Lemma 9.23. Since $\left.\Theta_{u}\right|_{W_{u}}$ is the standard deformation and as $\tau_{u} \in\left\langle J_{t, u}\right\rangle$ commutes with all elements in $J_{t, u}^{\perp}$ and with $r s r$ and $s$ (by Lemma $7.4 \mathrm{c})$ ), it follows that $\left.\delta_{u}\right|_{J \cup J_{t, u}^{\perp}} ^{\perp}=\left.\delta_{\infty}\right|_{J \cup J_{t, u}^{\perp}}$. Thus, it remains only to check whether $\delta_{u}(t)=\delta_{\infty}(t)$; but this is also a consequence of Lemma $\left.7.4 \mathrm{c}\right)$. This concludes the proof of the second assertion.

The last assertion is a consequence of the second, Lemma 9.23 and Proposition 3.3. 
Lemma 10.14 There exists an $(r, s, s r s)$-deformation $\delta$ of $S$ such that $\left.\delta\right|_{Y_{u}}=\delta_{u}$ for each $u \in \widehat{U}_{t}$.

Proof As $t$ is assumed to be wild, we have $\left|U_{t}\right| \geq 1$. We prove the lemma by induction on $\left|U_{t}\right|$. If $\left|U_{t}\right|=1$ and if $u$ denotes the unique element in $U_{t}$, then $S=Z_{u}$ and we are done by the previous lemma.

Suppose now $\left|U_{t}\right|>1$ and let $u \in U_{t}$. Put $C_{u}:=\bigcup_{u \neq u^{\prime} \in U_{t}} Z_{u^{\prime}}$. Note first that $C_{u} \cap Z_{u}=Y_{\infty}$ and that each edge in $S$ is contained in $C_{u}$ or in $Z_{u}$. By induction, there exists an $(r, s, s r s)$-deformation $\delta_{u}^{\prime}$ of $C_{u}$ such that $\left.\delta_{u}^{\prime}\right|_{Y_{u^{\prime}}}=\delta_{u^{\prime}}$ for each $u^{\prime} \in \widehat{U}_{t}$ different from $u$. By the previous lemma, there exists an $(r, s, s r s)$-deformation $\widehat{\delta}_{u}$ of $Z_{u}$ such that $\left.\hat{\delta}_{u}\right|_{Y_{a}}=\delta_{a}$ for $a \in\{u, \infty\}$. Now Proposition 3.3 yields the existence of $\delta$.

Conclusion of the proof of Theorem 10.4 The previous lemma yields the existence of an $(r, s, s r s)$-deformation $\delta$ of $S$ such that $\left.\delta\right|_{Y_{u}}=\delta_{u}$ for each $u \in \widehat{U}_{t}$. It remains to show that $\delta$ is tame. Let $t^{\prime} \in T$ be tame in $S$. Since $t$ is assumed to be wild, we have $t^{\prime} \neq t$. By Lemmas 9.22 and 9.16, there is an $u \in \widehat{U}_{t}$ such that $K_{t^{\prime}}^{\mathrm{def}}$ is contained in $Y_{u}$. By Lemma 10.13, we know that $\delta_{u}$ is a tame $(r, s, s r s)$-deformation. Hence there exists an element $v \in\left\langle Y_{u}\right\rangle$ such that $\left.\operatorname{Int}(v) \circ \delta_{u}\right|_{K_{t^{\prime}}^{\text {def }}}$ is the standard deformation of $K_{t^{\prime}}^{\text {def }}$. As $\left.\delta\right|_{Y_{u}}=\delta_{u}$, it follows that $\left.\operatorname{Int}(v) \circ \delta\right|_{K_{t^{\prime}}^{\text {def }}}$ is the standard deformation of $K_{t^{\prime}}^{\text {def }}$. Hence $\delta$ is tame.

\section{Proof of Theorem 2}

Let $(W, R)$ be a Coxeter system and let $S \subseteq R^{W}$ be a Coxeter generating set which is not sharp-angled. Suppose $S$ contains $k \geq 1$ edges which are not sharp-angled and choose one of them. Call it $J$. By Theorem 1, we can assume that $J=\{r, s\}$ with $o(r s)=5$. By Proposition 8.4, $J$ is a $\Delta$-edge. Hence, by Theorem 10.4, there exists a $J$-deformation $\delta$ of $S$ sending $J$ onto $\{r s r, s\}$. Hence, by Lemma $5.4, \delta(J)$ is a sharp-angled edge of $\delta(S)$. Let now $J^{\prime}$ be an edge of $S$ different from $J$. Then $\delta\left(J^{\prime}\right)$ is $W$-conjugate to $J^{\prime}$ by Property (AD4) of $\delta$; in particular, $\delta\left(J^{\prime}\right)$ is sharp-angled if and only if $J^{\prime}$ is sharp-angled. Hence the number of edges in $\delta(S)$ which are not sharp-angled is $k-1$. Thus the statement follows by an obvious induction on the number of edges of $S$ which are not sharp-angled.

\section{References}

[1] N Bourbaki, Éléments de mathématique. Fasc. XXXIV. Groupes et algèbres de Lie. Chapitre IV: Groupes de Coxeter et systèmes de Tits. Chapitre V: Groupes engendrés par 
des réflexions. Chapitre VI: systèmes de racines, Actualités Scientifiques et Industrielles 1337, Hermann, Paris (1968) MR0240238

[2] N Brady, J P McCammond, B Mühlherr, W D Neumann, Rigidity of Coxeter groups and Artin groups, from: "Proceedings of the Conference on Geometric and Combinatorial Group Theory, Part I (Haifa, 2000)", (L Mosher, M Sageev, editors), Geom. Dedicata 94 (2002) 91-109 MR1950875

[3] B Brink, R B Howlett, A finiteness property and an automatic structure for Coxeter groups, Math. Ann. 296 (1993) 179-190 MR1213378

[4] P-E Caprace, B Mühlherr, Reflection rigidity of 2-spherical Coxeter groups, Proc. Lond. Math. Soc. (3) 94 (2007) 520-542 MR2311001

[5] R Charney, M Davis, When is a Coxeter system determined by its Coxeter group?, J. London Math. Soc. (2) 61 (2000) 441-461 MR1760693

[6] V V Deodhar, A note on subgroups generated by reflections in Coxeter groups, Arch. Math. (Basel) 53 (1989) 543-546 MR1023969

[7] M Dyer, Reflection subgroups of Coxeter systems, J. Algebra 135 (1990) 57-73 MR1076077

[8] W N Franzsen, R B Howlett, Automorphisms of nearly finite Coxeter groups, Adv. Geom. 3 (2003) 301-338 MR1997410

[9] M Grassi, The isomorphism problem for a class of finitely generated Coxeter groups, $\mathrm{PhD}$ thesis, University of Sydney (2007)

[10] J E Humphreys, Reflection groups and Coxeter groups, Cambridge Studies in Advanced Math. 29, Cambridge University Press (1990) MR1066460

[11] B Mühlherr, The isomorphism problem for Coxeter groups, from: "The Coxeter legacy”, (C Davis, E W Ellers, editors), Amer. Math. Soc. (2006) 1-15 MR2209018

[12] J Ratcliffe, S Tschantz, Chordal Coxeter groups, Geom. Dedicata 136 (2008) 57-77

Université Libre de Bruxelles

Département de Mathématiques, Boulevard du Triomphe CP 216, Brussels, 1050, Belgium

Universität Giessen

Mathematisches Institut, Arndtstrasse 2, Giessen, D-35392, Germany

tmarquis@ulb.ac.be, bmuhlher@ulb.ac.be,

Bernhard.Muehlherr@math.uni-giessen.de

Received: 21 February 2008 Revised: 19 October 2008 